OPEN ACCESS

Edited by:

Sara Pedron,

University of Illinois at Urbana-Champaign,

United States

Reviewed by:

Ece Ozturk

Columbia University, United States

Joseph Chen,

University of California,

Berkeley, United States

Nor Eddine Sounni,

University of Liège, Belgium

*Correspondence:

Rachael W. Siriann rachael.sirianni@dignityhealth.org

Specialty section: This article was submitted to Biomaterials,

a section of the journa Frontiers in Materials

Received: 27 October 2017 Accepted: 26 January 2018 Published: 19 February 2018

Citation:

Heffernan JM and Sirianni RW (2018)

Modeling Microenvironmental Regulation of Glioblastoma Stem Cells: A Biomaterials Perspective.

Front. Mater. 5:7.

doi: 10.3389/fmats.2018.00007

\section{Modeling Microenvironmental Regulation of Glioblastoma Stem Cells: A Biomaterials Perspective}

\author{
John M. Heffernan ${ }^{1,2}$ and Rachael W. Sirianni ${ }^{1,2 *}$ \\ ${ }^{1}$ Laboratory for Nanomedicine, Barrow Brain Tumor Research Center, Barrow Neurological Institute (BNI), Phoenix, AZ, \\ United States, ${ }^{2}$ School of Biological and Health Systems Engineering, Arizona State University, Tempe, AZ, United States
}

Following diagnosis of a glioblastoma (GBM) brain tumor, surgical resection, chemotherapy, and radiation together yield a median patient survival of only 15 months. Importantly, standard treatments fail to address the dynamic regulation of the brain tumor microenvironment that actively supports tumor progression and treatment resistance. It is becoming increasingly recognized that specialized niches within the tumor microenvironment maintain a population of highly malignant glioblastoma stem-like cells (GSCs). GSCs are resistant to traditional chemotherapy and radiation therapy, suggesting that they may be responsible for the near universal rates of tumor recurrence and associated morbidity in GBM. Thus, disrupting microenvironmental support for GSCs could be critical to developing more effective GBM therapies. Three-dimensional culture models of the tumor microenvironment are powerful tools for identifying key biochemical and biophysical inputs that impact malignant behaviors. Such systems have been used effectively to identify conditions that regulate GSC proliferation, invasion, stem-specific phenotypes, and treatment resistance. Considering the significant role that GSC microenvironments play in regulating this tumorigenic subpopulation, these models may be essential for uncovering mechanisms that limit GSCs malignancy.

Keywords: tumor microenvironment, niche microenvironments, brain tumor stem cells, cancer stem cells, scaffolds, hydrogels, three-dimensional cell culture

\section{GLIOBLASTOMA (GBM)}

Glioblastoma is the most common and deadly pathological classification of malignant primary brain tumors. Epidemiological data collected for the United States between 2009 and 2013 indicate that GBM represents $46.6 \%$ of these diagnoses and $14.9 \%$ of all malignant and non-malignant primary brain tumor diagnoses (Ostrom et al., 2016). Overall age adjusted incidence rates are 3.2 per 100,000, with a median age of diagnosis of 64.0 years; risk rises with age (Ostrom et al., 2016). Symptoms of a GBM vary widely depending on tumor location and size but may include severe headaches, seizures, vision and speech impairment, or loss of cognitive and motor functions. Standard treatment modalities include removal of the bulk tumor via surgical resection, followed by radiotherapy and concomitant chemotherapy. However, treatment is rarely curative, and the prognosis is poor. Median survival remains stagnated at only 15 months (Stupp et al., 2009), and the 5-year survival rate is reported between 4.7 and 5.5\% (Omuro and DeAngelis, 2013; Ostrom et al., 2016). 


\section{Barriers to Treatment}

From a clinical perspective, successful treatment of GBM remains challenging due to several factors. Complete surgical resection, while the best treatment for GBM, is often impossible as a result of tumor location, as well as the potential for irreparable damage to healthy brain tissue during surgery (Sanai et al., 2011). Radiation treatment can often be targeted to areas of the brain that would be otherwise difficult to access surgically. Although radiation is an effective means for killing remaining tumor cells, simultaneous damage incurred on surrounding healthy tissue limits tolerability and may worsen patient outcome. Treatment of GBM with chemotherapeutics is inhibited by the blood-brain barrier, which segregates the brain from systemic circulation and prevents the vast majority of drugs from effectively reaching malignant cells in the brain. The primary chemotherapeutic currently used in GBM treatment is the DNA alkylating agent temozolomide, which is administered orally, is brain available and generally well tolerated, but imparts only a modest improvement in patient outcome (Stupp et al., 2009). Overall, current treatment options remain inadequate.

One of the key biological features of GBM is that, unlike other tumor types tumors, it does not metastasize through the blood to peripheral organs; instead, individual cells invade healthy brain, preferentially migrating along white matter tracts and perivascular spaces (Giese and Westphal, 1996; Giese et al., 2003). These cells are responsible for initiating secondary tumors that most often arise within centimeters of the original tumor (Petrecca et al., 2012) but may manifest even on the contralateral side of the brain (Matsukado et al., 1961; Giese and Westphal, 1996). Invasive cells are undetectable by current imaging methods, and almost impossible to remove via surgical resection without damaging healthy brain. Radiation and chemotherapy fail to address invasive cells that are shielded by radiosensitive healthy tissue and an intact blood-brain barrier. Thus, the invasive nature of GBM drives near universal rates of tumor recurrence as secondary tumors arise from seemingly healthy brain (Petrecca et al., 2012; Omuro and DeAngelis, 2013).

A factor that further complicates the treatment landscape is that GBM tumors display a high degree of genetic, epigenetic, and cellular diversity. Presently, GBM is classified into four distinct subtypes: Proneural, Neural, Classical, and Mesenchymal; each of which corresponds to a common set of neoplastic genetic alterations (Verhaak et al., 2010). However, individual subtype classifications may not be relevant to all cells found in a single tumor, as intratumoral heterogeneity is also a common feature of GBM (Sottoriva et al., 2013). This heterogeneity is a primary source of treatment resistance, whereby one tumor region that is sensitive to treatment is sustained or replaced by another region that is tolerant (Bonavia et al., 2011). Tumor heterogeneity is therefore an adaptive growth pattern that is challenging to address through monotherapy, even when targeted.

\section{GBM TUMOR MICROENVIRONMENT}

The GBM tumor microenvironment is a complex regulatory structure composed of cellular and non-cellular components that collectively contribute to disease progression. The tumor microenvironment significantly influences many important aspects of GBM biology; the specific functions of the GBM tumor microenvironment have been reviewed extensively by others (Bonavia et al., 2011; Charles et al., 2011; Wiranowska and Rojiani, 2011; Xiao et al., 2017); here, we provide an overview to introduce key features of this relationship that are important for modeling this unique microenvironment.

Unlike other tissues in the body, the brain extracellular matrix (ECM) does not contain high concentrations of fibrous proteins (Bellail et al., 2004). Instead, the dense tumor microenvironment ECM is primarily composed of the glycosaminoglycan hyaluronic acid (HA), and to a lesser degree, tenascin-C, collagen IV and V, fibronectin, laminin, and vitronectin, which are generally associated with blood vessels (Delpech et al., 1993; Giese and Westphal, 1996; Bellail et al., 2004; Wiranowska and Rojiani, 2011; Rape et al., 2014). GBM cells interact with HA via the cell surface receptors CD44 and RHAMM, which are often overexpressed on GBM cells, and promote invasive phenotypes (Merzak et al., 1994; Ariza et al., 1995; Koochekpour et al., 1995; Akiyama et al., 2001). The proteoglycan tenascin- $\mathrm{C}$ has been described to promote GBM invasion (Giese and Westphal, 1996; Sarkar et al., 2006) and to stimulate angiogenesis, which leads to tumor progression (Bellail et al., 2004; Rape et al., 2014). Other vascular-associated ECM constituents (collagen, fibronectin, laminin, and vitronectin) both promote and guide GBM invasion into healthy brain tissue (Mahesparan et al., 2003; Kawataki et al., 2007; Rape et al., 2014). Moreover, these molecules also enable integrin-mediated focal adhesions, which play a significant role in GBM progression, and have been proposed as a biomarker target for treatment (Kawataki et al., 2007; Lathia et al., 2010; Ruiz-Ontañon et al., 2013; Paolillo et al., 2016; Haas et al., 2017). Furthermore, in addition to affecting invasion, various isoforms of laminin proteins have been shown to potentiate glioblastoma stem-like cell (GSC) phenotypes via integrin interactions (Pollard et al., 2009; Lathia et al., 2010, 2012; Haas et al., 2017).

The concentration of ECM in the tumor microenvironment is increased compared with healthy brain, as its constituents are manufactured by GBM cells to support tumor progression (Delpech et al., 1993; Giese and Westphal, 1996; Akiyama et al., 2001; Wiranowska and Rojiani, 2011; Lathia et al., 2012). The dense ECM and the high cellularity of the tumor contribute to increased mechanical stiffness. GBM cells sense microenvironmental stiffness primarily through integrins and focal adhesion complexes via mechanosensation (Rape et al., 2014). While the biochemical and biophysical effects of matrix composition appear to be complementary or even synergistic in promoting malignancy, they are not easily isolated in vivo. In vitro studies have identified stiffness as a strong regulator of GBM proliferation and invasion (Ananthanarayanan et al., 2011; Wiranowska and Rojiani, 2011; Pathak and Kumar, 2012; Pedron and Harley, 2013; Heffernan et al., 2014; Kim and Kumar, 2014; Rape et al., 2014; Umesh et al., 2014). Moreover, blood vessels provide the greatest stiffness in the brain and are preferential routes for GBM invasion (Giese and Westphal, 1996; Giese et al., 2003). Therefore, it is likely that the stiffness of these and other structures instructs malignant behaviors in vivo. 
The tumor-associated cells within the tumor microenvironment are key regulators of GBM growth and tumor vascularization. Cells that commonly provide support to GBM include tumor-associated endothelial cells, pericytes, astrocytes, fibroblasts, and infiltrating immune cells such as macrophages and microglia (Charles et al., 2011). One of the primary modes of support from tumor and tumor-associated cells is secretion of soluble signaling factors that stimulate malignant phenotypes, i.e., proliferation (EGF, FGF, IGF, and HGF), angiogenesis [vascular endothelial growth factor (VEGF)], and invasion (IGF, HGF, and TGF- $\beta$ ) (Wiranowska and Plaas, 2008). These secreted signaling factors may be sequestered within the dense network of ECM and serve as a depot for GBM cells (Wiranowska and Plaas, 2008; Wiranowska and Rojiani, 2011). Tumor vascularization is achieved in part by recruitment of vascular-associated endothelial cells, pericytes, and astrocytes, to meet the nutrient demands of a growing tumor (Wiranowska and Plaas, 2008; Charles et al., 2011; Soda et al., 2013). Specifically, endothelial cells are stimulated to proliferate and migrate toward tumor regions with poor oxygenation as a result of VEGF productions by hypoxic tumor cells (Soda et al., 2013). However, the resulting tumor-associated neovasculature is significantly different from healthy vessels as it forms a dense and disordered network of leaky vessels with necrosis developing in regions of severe chronic hypoxia $(<1 \%$ $\mathrm{O}_{2}$ ) (Holmquist-Mengelbier et al., 2006; Soda et al., 2013).

\section{GLIOBLASTOMA STEM-LIKE CELLS}

Before 2003, GBM, like most solid tumors, was widely believed to be driven by a stochastic model of clonal evolution in which tumors were initiated via neoplastic transformation of glia. The identification and characterization of tumorigenic GSCs within human brain tumors have since reshaped conventional wisdom over the architecture of GBM biology (Ignatova et al., 2002; Singh et al., 2003, 2004; Galli et al., 2004). This discovery supports the hypothesis that cells within a tumor display a hierarchical order of tumorigenic potential that is maintained by cancer stem cells (Tan et al., 2006; Venere et al., 2011). It is now widely recognized that within GBM tumors, GSCs are essential to tumor maintenance, drivers of heterogeneity, and also may represent the cell of origin (Sanai et al., 2005; Venere et al., 2011).

\section{GSC Characteristics}

Glioblastoma stem-like cells display many biological similarities to neural stem cells (NSCs); they are capable of indefinite selfrenewal and multipotent differentiation, and they express genes that promote NSC phenotypes such as NESTIN, SOX2, and OLIG2 (Ignatova et al., 2002; Singh et al., 2003, 2004; Galli et al., 2004; Sanai et al., 2005; Lee et al., 2006; Ligon et al., 2007). Identification and enrichment of GSCs can be achieved by sorting tumor cells that express validated cell surface biomarkers [CD133 (Singh et al., 2003), SSEA-1 (Son et al., 2009), and Integrin $\alpha 6$ (Lathia et al., 2010)] followed by functional analysis of stem behaviors (Lathia et al., 2015). Of the stem behaviors, none is more important to GSC tumorigenicity than self-renewal. This was demonstrated when GSCs were first isolated and were observed to form orthotopic xenograft tumors from as few as
100 cells. In comparison, non-stem GBM cells (NGSCs) from the same tumor sample were incapable of forming tumors from injections of 100,000 cells (Singh et al., 2004). GSCs also display a capacity for multipotent differentiation into non-tumorigenic cancer-associated cells, such as vascular cells, that provide critical support for tumor growth (Ricci-Vitiani et al., 2010; Wang et al., 2010a; Lathia et al., 2011; Cheng et al., 2013a). Multipotency contributes to cellular heterogeneity observed in primary GBM; this behavior has been recapitulated in experimental orthotopic xenograft tumor models (Singh et al., 2004).

Ex vivo purification of GSCs requires a multistep process that tests self-renewal, multipotency, and stem-marker expression. Failure to test all three components often results in false positive identification (Lathia et al., 2015). Another method for identifying GSCs has been through the use of label retaining assays to identify quiescent or slow-cycling cells (Deleyrolle et al., 2011; Zeng et al., 2016). Using robust verification, long-term established GBM cell lines are found to lack fully functional GSCs, even in NSC culture conditions (Lee et al., 2006; Lathia et al., 2015). Thus to properly research GSC behaviors, experiments should ideally be performed on low-passage patient-derived cells that have been validated as a stem population.

\section{GSC Response to Treatment}

Glioblastoma stem-like cells are highly treatment resistant, which is facilitated by their propensity to invade healthy brain (Cheng et al., 2011), potential quiescence (Chen et al., 2012), and activation of molecular machinery that is protective against radiation (Bao et al., 2006a) and cytotoxic insult (Liu et al., 2006). Many of the invasive mechanisms utilized by GSCs mimic NSC motility along white matter tracts and blood vessels (Sanai et al., 2005). Thus, GBM tumors characteristically display an infiltrative leading edge that disseminates into healthy tissue. GSC derived orthotopic xenograft tumors recapitulate this invasive behavior with GSCs concentrated at the tumor edge (Strojnik et al., 2007), whereas NGSCs from the same patient tumor sample are minimally invasive (Cheng et al., 2011). Resistance to both radiation (Bao et al., 2006a) as well as many conventional chemotherapeutics, including temozolomide (Liu et al., 2006; Chen et al., 2012), has been reported in the GSC population. This resistance is ascribed to increased activation of DNA damage checkpoint and repair proteins (Bao et al., 2006a), as well as increased expression of ATP-binding cassette drug transporters, which contribute to increased drug efflux and chemoresistance (Bleau et al., 2009). Recurrent tumors are also enriched for GSCs compared with the primary tumor suggesting that GSCs evade conventional therapy and play a prominent role in the high rates of GBM relapse (Liu et al., 2006).

\section{GSC NICHE MICROENVIRONMENTS}

Similar to NSCs, which are primarily found in the subventricular zone and hippocampus of the adult brain, GSCs are also concentrated in niche microenvironments (Figure 1) (Sanai et al., 2005). One notable difference is that GSC niche microenvironments appear to be mitogenic, encouraging growth, while NSCs are generally sustained in quiescence (Lathia et al., 2011). These 

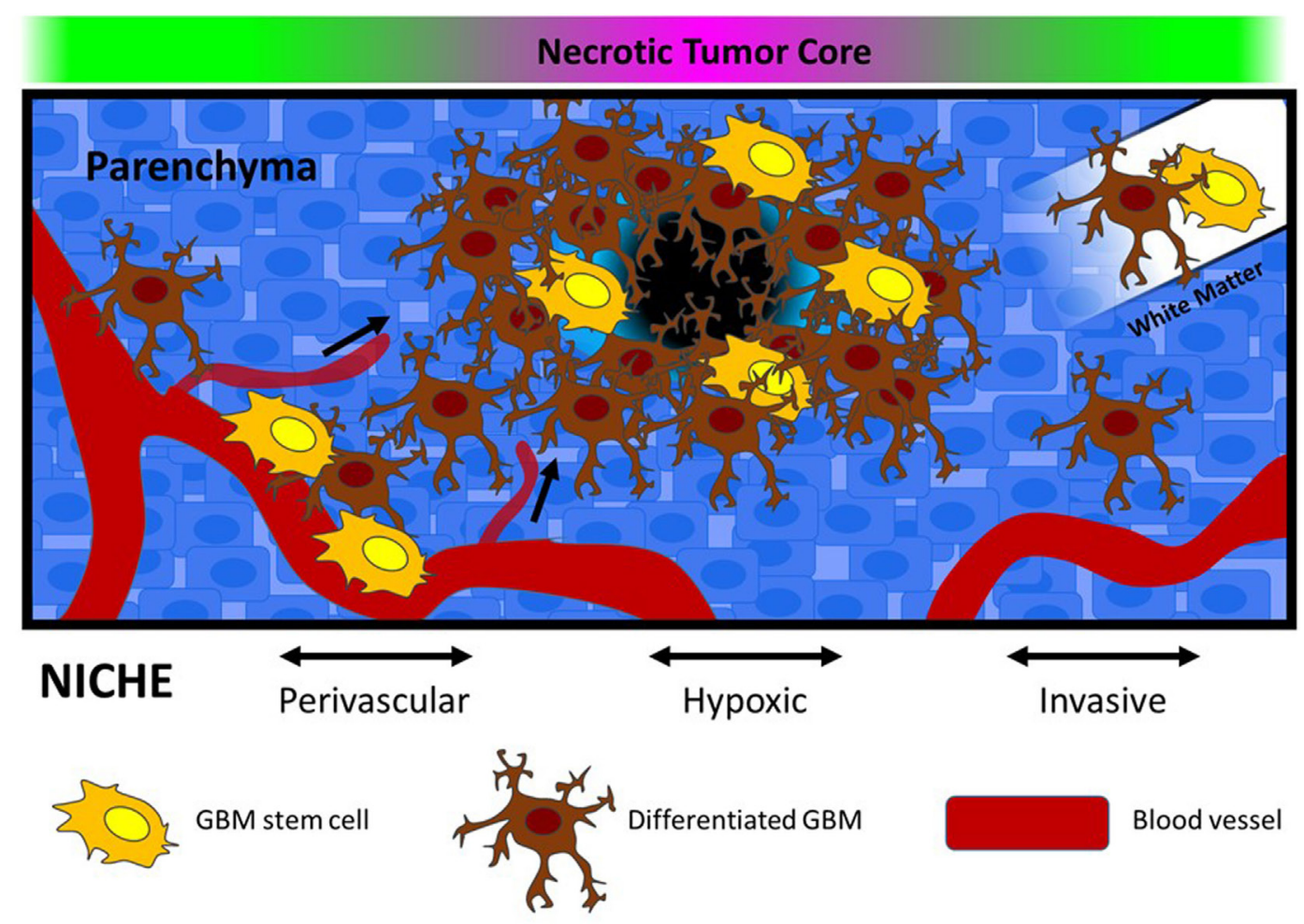

Blood vessel

FIGURE 1 | Glioblastoma stem-like cells are located in defined microenvironments within the brain, including perivascular, hypoxic, and invasive niches. Each niche varies in terms of biophysical features (stiffness and porosity), extracellular matrix composition, and oxygen availability. In the graphic, vessel co-option is depicted within the perivascular niche, and movement along white matter tracts is depicted in the invasive niche. Within the hypoxic niche, necrosis and altered metabolism is expected to drive alterations in $\mathrm{pH}$, as well as recruitment of new blood vessels.

physical regions within the larger tumor microenvironment include a range of microenvironmental features that sustain and regulate GSC phenotypes through hypoxia, growth factor signaling, and adhesion to the ECM. It is thus unsurprising that the microenvironment plays a role in provoking treatment resistance (Gilbertson and Rich, 2007; Rich, 2007; Jamal et al., 2010, 2012; Mannino and Chalmers, 2011; Lathia et al., 2012).

\section{Perivascular Niche}

A niche microenvironment has been identified in regions directly adjacent to blood vessels known as the vascular or perivascular niche (Calabrese et al., 2007; Gilbertson and Rich, 2007). Tumor vascularization is a requisite process to provide GBM tumors with adequate oxygen and nutrients that sustain rapid growth. Bao et al. determined that GSCs initiate neovascularization by stimulating endothelial cell proliferation, migration, and tube formation through secretion of VEGF and stromal-derived factor 1 (Bao et al., 2006b; Folkins et al., 2009). In parallel, vascular endothelial cells promote GSC self-renewal and proliferation, through secretion of soluble signaling factors such as nitric oxide, as well as via activation of NOTCH signaling (Calabrese et al., 2007; Charles et al., 2010; Hovinga et al., 2010; Galan-Moya et al., 2011). This support appears to be unique to endothelial cells. For example, Calabrese et al. (2007) determined that neither NGSCs, astrocytes, nor fibroblasts were able to produce comparable enrichment of GSCs in vitro. Importantly, GSCs are also capable of transdifferentiation into tumor-derived vascular cells. In experimental tumor models, GSCs have been observed to differentiate into pericytes and endothelial cells that participate in the formation and maintenance of neovasculature (Ricci-Vitiani et al., 2010; Wang et al., 2010a; Lathia et al., 2011; Cheng et al., 2013a; Schonberg et al., 2014). Therefore, interactions between endothelial cells and GSCs in the perivascular niche may create a self-sustaining paracrine signaling cycle that is critical for tumor maintenance and progression (Schonberg et al., 2014).

\section{Hypoxic Niche}

In juxtaposition to the nutrient-rich perivascular niche, GSCs are also found concentrated surrounding tumor regions that have limited access to blood vessels and are often necrotic (Li et al., 2009). The disorganized vasculature of GBM tumors leads to regional oxygen concentration gradients that have significant effects on GSC phenotypes. The primary molecular response to oxygen deprivation involves activation of the hypoxia inducible factor (HIF) family of transcription factors whose canonical downstream targets are proangiogenic (Heddleston et al., 2010). As a result, the hypoxic niche may in some cases exist as a transitional microenvironment in which GSCs use proangiogenic factors such as VEGF to recruit blood vessels and establish a perivascular niche (Venere et al., 2011). 
Hypoxia inducible factor activation has also been found to be a potent regulator of various GSC behaviors. HIF1 $\alpha$ and HIF2 $\alpha$ exhibit overlapping functions in both vasculogenesis and enriching stem phenotypes (Heddleston et al., 2009, 2010; Li et al., 2009; Soeda et al., 2009; Bar et al., 2010; Seidel et al., 2010). However, unique downstream target genes have also been identified particularly for HIF2 $\alpha$, which include stem markers Oct4, c-Myc, and Nanog (Heddleston et al., 2009; Li et al., 2009; Keith et al., 2011). Importantly, Li et al. (2009) reported that while HIF1 $\alpha$ is expressed in both NSC and GSC populations, HIF $2 \alpha$ expression is restricted to GSCs and is required for GSC tumorigenicity in vivo. HIF $2 \alpha$ induction also promotes stem plasticity in the pool of NGSCs, which may be particularly important for repopulating the GSC pool in response to treatment (Heddleston et al., 2009). HIF expression also appears to be regulated by distinct components of the microenvironment. HIF $1 \alpha$ expressing cells are enriched in regions of chronic hypoxia $\left(>1 \% \mathrm{O}_{2}\right)$, while HIF $2 \alpha$ expression is more sporadically identified in both hypoxic and normoxic regions surrounding blood vessels (Holmquist-Mengelbier et al., 2006; Li et al., 2009). In addition, tumor acidity, a byproduct of overactive glycolytic energy production, increases HIF $2 \alpha$ stabilization independent of oxygen concentration and also promotes stem plasticity (Hjelmeland et al., 2011). Thus, the hypoxic niche regulates GSC phenotypes primarily through HIF activity, which is essential to stem maintenance and tumorigenicity.

\section{Invasive Niche}

Glioblastoma stem-like cell populations have been identified at the leading edge of GBM tumors suggesting that this invasive front also contributes to GSC maintenance (Lee et al., 2006; Kitai et al., 2010; Cheng et al., 2011; Ishiwata et al., 2011; Lathia et al., 2011; Ortensi et al., 2013). Orthotopic GSC tumors recapitulate the invasive profile observed in patient tumors compared with NGSCs, which generally form noninvasive tumors (Cheng et al., 2011). Furthermore, recurrent tumors are enriched with GSCs indicating that these cells are likely responsible for infiltrative growth that is characteristic of GBM (Liu et al., 2006). Therefore, while an invasive niche has yet to be definitively established, microenvironmental interactions, particularly with the ECM protein laminin, have been identified that regulate both invasive behaviors and stem phenotypes. For example, laminin receptor integrins $\alpha 6$ and $\alpha 7$ have been proposed as biomarkers for functional GSCs (Lathia et al., 2010; Haas et al., 2017), while GSC regulation has also been described through interactions with the laminin subunit $\alpha 2$ (Lathia et al., 2012). In healthy brain, laminin is primarily located on the outside of blood vessels, which are primary routes of GBM invasion (Giese and Westphal, 1996). Expression of laminin (Ljubimova et al., 2006) and localization of laminin within the perivascular niche (Lathia et al., 2012) relate to poor patient prognosis; the integrin family of laminin receptor proteins are overexpressed in CD133 + GSCs, which have been demonstrated to promote invasion (Nakada et al., 2013), proliferation (Lathia et al., 2012), and resistance to apoptosis (Huang et al., 2012). These integrin-mediated phenotypic shifts are significant at the level of disease progression and may be related to active microenvironmental regulation (Paolillo et al., 2016). For example, Ljubimova et al. (2006) observed a switch in laminin isoform expression during tumor progression that was associated with both invasion and angiogenesis, suggesting that laminin is a dynamic partner in the development of tumor malignancy. The interaction of tumor cells with vascular-associated laminins has been shown to be an important factor for GSC regulation in the perivascular niche (Lathia et al., 2012). In addition, certain isoforms of laminin sustain GSC phenotypes during in vitro stem cell cultures (Pollard et al., 2009), and conversely, may also be used to promote GSC differentiation to NGSCs in serum-supplemented conditions (Ignatova et al., 2002). These relationships warrant further study to understand the complexities of GBM-laminin interactions, and to determine how they may contribute to treatment resistance and/or tumor recurrence.

\section{Therapeutic Challenges and Opportunities}

Along with regulatory inputs, niche microenvironments provide GSCs with protection from cytotoxic treatments (Gilbertson and Rich, 2007). The perivascular niche has been described as radioprotective for medulloblastoma tumors (Hambardzumyan et al., 2008). This resistance was initiated by signaling through the oncogenic PI3K/Akt pathway, which is a downstream target of the epidermal growth factor receptor (EGFR). In relation to GBM biology, EGFR is one of the most important biomarkers for malignancy (Verhaak et al., 2010) and is critical to the maintenance of stem phenotypes in vitro (Lee et al., 2006). Moreover, inhibiting EGFR has been observed to sensitize otherwise radioresistant GSCs to treatment (Kang et al., 2012). Therefore, activation of this receptor in the nutrient-rich perivascular niche would conceivably negatively impact the efficacy of radiotherapy on GSCs. VEGF signaling, which is critical for tumor vascularization and establishment of the perivascular niche, has also been shown to enhance resistance to radiation (Knizetova et al., 2008). Similarly, Notch signaling, which functions through direct cell-cell contact of transmembrane proteins, also supports radioresistant behaviors in GSCs and is an integral signaling pathway in the vascular niche (Wang et al., 2010b).

The hypoxic niche provides some of the best direct evidence of niche protection from chemotherapy and radiation. For both treatments, a common mode of action is through the generation of reactive oxygen species (ROS), which induces double strand breaks in DNA (Harrison and Blackwell, 2004). However, due to the relative lack of oxygen, ROS generation is attenuated thereby limiting this mechanism. In addition, hypoxia is capable of promoting downstream activation of numerous survival pathways that may further limit treatment efficacy (Harrison and Blackwell, 2004; Bhatt et al., 2008; Bertout et al., 2009). For example, GSCs identified in hypoxia have been observed to highly express MGMT, which functions to repair DNA and promotes resistance to TMZ (Pistollato et al., 2010).

Targeting niche microenvironments may provide an opportunity to disrupt GSC regulation and increase GBM treatment efficacy. Recently, inhibition of vascular niche formation initially appeared to be a promising direction for the development of new treatments; in experimental tumors, GSCs were depleted and tumor growth retarded by the antiangiogenic therapy bevacizumab, which is a VEGF function blocking antibody (Bao et al., 2006b; Calabrese et al., 2007). However, bevacizumab was 
subsequently found to effect an increase invasion of GBM cells in response to increased hypoxia resulting from the inhibition of blood vessel formation (Pàez-Ribes et al., 2009; Keunen et al., 2011). In a phase III clinical trial, this treatment failed as a first-line therapy but remains an approved and viable option as a salvage treatment for increasing progression free survival in recurrent GBM (Kreisl et al., 2008). Bevacizumab fails as a GBM treatment primarily as a result of the strong hypoxia response of these tumors, and thus any approach seeking to inhibit blood supply to GBM must consider molecular responses of cells to hypoxic environments. For example, HIF $2 \alpha$ may present a potential cotherapeutic target due to its specificity for GSCs, prominent role in GSC tumorigenicity, and regulation of responses to oxygen ( $\mathrm{Li}$ et al., 2009; Hjelmeland et al., 2011).

Glioblastoma stem-like cell niches are complex and diverse microenvironments that provide adaptive regulation of stem functions along with protective support against GBM treatments. The striking capacity of these cells to survive insults decreases the likelihood that any monotherapy will be significantly effective. Therefore, although clinical results have thus far been disappointing, targeting and disrupting microenvironmental mechanisms of GSC regulation should remain a focus of novel treatment designs.

\section{GBM RESEARCH MODELS}

\section{Cell Lines and In Vitro Culture}

In vitro cell culture models have been fundamental to GBM research since the first tumor cell lines were isolated and immortalized in the 1960s (Ponten and Macintyre, 1968). Various immortalized cell lines are now widely available for research and provide a platform for disease research that ideally enables reproducible testing. Propagation in vitro is performed using a simplified two-dimensional (2D) isotropic plate [often poly(styrene)] that is treated to present a negative charge, or coated with poly-D-lysine, or ECM proteins to promote anchorage dependent cell growth. This $2 \mathrm{D}$ design is optimized for cells to experience consistency in their access to adhesion sites, nutrients, soluble signaling factors, and oxygen in culture (Woolard and Fine, 2009). These cultures enable biologically instructive assays that measure behaviors such as proliferation, migration, stem cell status, and drug sensitivity under various discrete conditions (Giese et al., 1995; Pollard et al., 2009).

Although immortalized GBM cell lines have provided invaluable understanding of aspects of the disease process in GBM, their utility in generating new therapies for clinical application is limited. In vitro, cells are polarized and attach to the stiff culture substrate in a single plane that provides little to no resistance to proliferation or migration. In response, cells converge on a singular phenotype through a rapid loss of cellular heterogeneity, which is a fundamental feature of GBM (Li et al., 2008). Immortalized cell lines show significant differences in their molecular signature compared with primary GBM tissue, which is a direct result of prolonged propagation and genetic instability (Li et al., 2008). Another prominent issue with long-term cell lines is the potential for contamination with other cell lines that replace the original population. As an example, one of the most widely used and first established in vitro models of GBM, the U87 MG cell line, was originally isolated from a 44-year-old female patient (Ponten and Macintyre, 1968). Recently, the genome of this line was compared with the original tumor sample and was determined to be a GBM of male origin (Allen et al., 2016). These problems, among others, illustrate that more representative disease models are necessary to overcome challenges in studying GBM biology.

The use of low-passage primary cells derived from patient tissue provides an improvement in the biological relevance of in vitro models. These are established by mechanical and enzymatic digestion of tumor tissue, whereby the resulting heterogeneous cell mix is cultured in vitro using standard culture conditions. GSC lines may also be established from primary tissue through culture in serum-free NSC optimized media with the mitogenic growth factors EGF and FGF (Venugopal et al., 2012). These conditions maintain the GSC population such that cells preserve genotypic and phenotypic features of the original tumor, whereas serum-supplemented cultures promote selection of differentiated GBM phenotypes and the GSC pool is subsequently depleted (Zhang et al., 2013; Lathia et al., 2015).

Standard 2D cultures may also be modified to produce models that better represent native GBM biology. For example, GBM has been cocultured with a secondary cell type such as astrocytes (Rath et al., 2013, 2015) or endothelial cells (Galan-Moya et al., 2011) to promote malignant phenotypes. The most common method for establishing cocultures is through a transwell or Boyden chamber system. In these cultures, cells are separated by a semi-permeable membrane that allows access to signaling factors secreted by the otherwise physically separated cell populations. Coculture studies have indicated that supporting cells are well capable of directing the behavior of tumor cells, including provocation of invasion and treatment resistance (Galan-Moya et al., 2011; Rath et al., 2013, 2015).

Similar to coculture methods, three-dimensional (3D) cell cultures model aspects of the tumor microenvironment to elicit interactions that are generally absent from $2 \mathrm{D}$ cultures. Techniques such as hanging drop culture or culture on soft agar gels generate multicellular GBM spheroids that exhibit proliferation and invasion that better recapitulates in vivo scenarios (Del Duca et al., 2004; Pampaloni et al., 2007; Heffernan et al., 2014). Suspension culture, in which non-adherent cells are free-floating in media, is most often used to propagate GSCs where, similar to NSCs, stem-like cells form multicellular neurospheres (also called tumorspheres) (Ignatova et al., 2002; Singh et al., 2003; Galli et al., 2004; Lee et al., 2006; Fael Al-Mayhani et al., 2009; Venugopal et al., 2012). Spheroid cultures can also be initiated as cocultures in which GBM cells are combined with endothelial or glial cells and incorporated into spheroid structures (Chen et al., 2009). Brain slice cultures further improve the relevance of the in vitro culture by enabling GBM cells to be analyzed in live brain tissue ex vivo. Here, viable brain slices are cultured and inoculated with tumor cells to enable tracking of GBM proliferation and invasion within a complete brain microenvironment. The primary drawbacks to brain slice culture include technical challenges with maintaining the tissue, reproducibility, and rapid cell death and/or alterations in the tissue during cultures (Rao et al., 
2014; Jensen et al., 2016). Taken in sum, each of these approaches to culturing GBM have been valuable to isolate specific cellular responses under defined experimental conditions, although the degree to which neurosphere or hanging drop cultures can be engineered to capture essential aspects of the niche remain limited.

\section{Preclinical In Vivo Models}

In vivo models of GBM are the gold standard for analyzing tumor growth and response to therapy within a physiologically relevant system. In vivo models are either syngeneic or xenograft. Syngeneic murine GBM models have been established via development of native GBM cell lines (e.g., through chemical insult) or genetic engineering that induces spontaneous and reproducible tumor formation (Huszthy et al., 2012). Primary advantages of syngeneic models include the ability to analyze tumors in the context of a fully functional immune system, and in genetic models, alterations in signaling pathways that are known to drive GBM malignancy (EGFR, PDGFR, Rb, Ras, and Akt) (Jacobs et al., 2011; Huszthy et al., 2012). Alternatively, xenograft models are established by the transplantation of human derived cell lines into an immunocompromised mouse host. The primary advantage of xenograft models is that they enable study of human GBM progression within a functional albeit immunodeficient brain.

Human xenograft models may be established from longterm cell lines or from freshly isolated patient-derived GBM tissue. Tumors can be induced in either the flank or directly in the brain. Flank models enable rapid confirmation of tumorigenicity and rapid growth of tumor within an easy to access physical compartment, while also providing a more permissive paradigm for treatment studies due to the lack of a blood-brain barrier protecting the tumor. Orthotopic models, on the other hand, are best suited for studying GBM behaviors in the context of the native brain tumor microenvironment. Various immortalized cell lines (U87, U118, etc.) have been used to produce aggressive orthotopic tumors with reproducible cellular architecture (Jacobs et al., 2011; Huszthy et al., 2012). However, tumors generated through orthotopic transplant of immortalized cells often present significant genetic and histological variations from patient tumors thereby limiting their translational relevance (Lee et al., 2006; Jacobs et al., 2011). For example, U87 tumors are highly vascularized, possess a relatively leaky blood-brain barrier, and do not exhibit the infiltrative behavior that is characteristic of patient GBM tissue (Jacobs et al., 2011). The generation of noninvasive tumors is one of the primary drawbacks common to using immortalized cells in preclinical models. Conversely, low-passage patient-derived xenografts, particularly those established in serum-free culture or via direct in vivo inoculation, are characterized by their maintenance of parental tumor genotypes, an invasive leading edge, and minimal disruption of the blood-brain barrier (Galli et al., 2004; Singh et al., 2004; Sanai et al., 2005; Lee et al., 2006; Fael Al-Mayhani et al., 2009). Thus, patient-derived xenografts are presently considered the most biologically relevant research model of the human disease (Huszthy et al., 2012).

\section{ENGINEERING THE GBM TUMOR MICROENVIRONMENT}

The reduction of microenvironmental complexity in $2 \mathrm{D}$ cell culture limits analysis of disease biology because the 3D ECM regulates numerous essential cellular phenotypes (Pampaloni et al., 2007). Tissue engineering strategies address this gap in understanding by providing methods to model key components of the $3 \mathrm{D}$ tumor microenvironment such as insoluble ECM components, stiffness, matrix degradability, and soluble signaling factors. These tools are not a direct surrogate for the complex, anisotropic, and heterogeneous in vivo scenario; instead, they enable characterization of contributions from individual microenvironmental factors. Here, we review how these approaches have been utilized to understand important features of GBM and GSC biology.

\section{Biomaterials in GBM Research}

Both natural and synthetic polymers have been used to study GBM response to the microenvironment (relevant studies are summarized in Table 1). Natural materials are bioactive, degradable by enzymatic or hydrolytic mechanisms, and cells interact with them directly through specific and established biochemical pathways. Due to their specific bioactivity, some of the most important and commonly used in GBM research are HA, collagen, and Matrigel ${ }^{\circledR}$ (Table 1). One potential challenge with using ECM biomaterials derived from live hosts or cell cultures, such as Matrigel ${ }^{\circledR}$, is a lack of experimental reproducibility. These multicomponent materials exhibit variation in composition across batches (e.g., growth factor content and ECM protein concentration), which may adversely impact the interpretation of results due to changes in the constituent materials (Pampaloni et al., 2007). Juxtaposed to natural materials, synthetic biomaterials used in GBM cultures are derived from organic sources, which enables a high degree of control over their physical and chemical properties. Of these, poly(ethylene glycol) (PEG) is by far the most common. Its hydrophilicity and chemical structure enable cell encapsulation and functionalization reactions that can be performed in situ. Synthetic biomaterials can be either degradable or non-degradable, and in general, are expected to possess lower intrinsic bioactivity than natural materials, since they do not possess cellular adhesion sites that would be expected to elicit biological responses. Cells are capable of interfacing with a purely synthetic polymer either through surface adsorbed proteins (vitronectin, laminin, etc.) or through non-specific charge interactions (Hubbell, 1995). Grafting synthetic polymers with bioactive proteins or peptides (e.g., RGD) is a common approach to enable cellular adhesion or biodegradation (Table 1). This method of combining natural and/or synthetic components into a composite biomaterial is useful for leveraging advantages of both classifications.

The majority of natural, synthetic, and composite scaffolds applied in GBM studies are hydrophilic hydrogels, which, like tissue, are composed of a high fraction of water and swell considerably in aqueous solution. However, in some instances hydrophobic polymers are also incorporated, often coated with 
TABLE 1 | Biomaterial models of the glioblastoma (GBM) microenvironment enable analysis of malignant behaviors in vitro.

\section{Reference}

\section{SCAFFOLD COMPONENTS}

Hyaluronic acid

Tamaki et al. (1997), Jin et al. (2009), Ananthanarayanan et al. (2011), Florczyk et al. (2013), Pedron et al. (2013, 2015, 2017), Rao et al. (2013a,b), Fernandez-Fuente et al. (2014), Heffernan et al. (2014), Jiguet Jiglaire et al. (2014), Kim and Kumar (2014), Rape and Kumar (2014), Wang et al. (2014, 2016), Herrera-Perez et al. (2015), Rape et al. (2015), Cha et al. (2016), Kievit et al. (2016), Chen et al. (2017), and Ngo and Harley (2017)

Collagen Tamaki et al. (1997), Sarkar et al. (2006), Kim et al. (2008), Ulrich et al. (2010), Yang et al. (2010, 2014), Eke et al. (2012), Florczyk et al. (2013), Pedron and Harley (2013), Pedron et al. (2013, 2015, 2017), Rao et al. (2013a,b), Ruiz-Ontañon et al. (2013), Fernandez-Fuente et al. (2014), Heffernan et al. (2014), Jain et al. (2014), Jiguet Jiglaire et al. (2014), Herrera-Perez et al. (2015), Wong et al. (2015), Cha et al. (2016), Chen et al. (2017), Chonan et al. (2017), and Ngo and Harley (2017)

Matrigel Cordes et al. (2003), Jin et al. (2009), Kievit et al. (2010), Cheng et al. (2011), Rao et al. (2013a), Ruiz-Ontañon et al. (2013), HerreraPerez et al. (2015), Grundy et al. (2016), Hubert et al. (2016), and Chonan et al. (2017)

Poly(ethylene glycol) Pedron and Harley (2013), Pedron et al. (2013), Heffernan et al. (2014), Jiguet Jiglaire et al. (2014), Wang et al. (2014), Fan et al. (2016), Li et al. (2016), Oh et al. (2016), and Ngo and Harley (2017)

Chitosan Kievit et al. (2010, 2014, 2016), Florczyk et al. (2013, 2016), and Wang et al. (2016)

Alginate Kievit et al. (2010, 2014, 2016), Florczyk et al. (2016), and Oh et al. (2016)

Poly(N-isopropylacrylamide)

Polyacrylamide Heffernan et al. (2016, 2017), and Li et al. (2016)

\begin{tabular}{l} 
Polyacrylamide \\
\hline Polycaprolactone \\
\hline Polystyrene \\
\hline Poly(lactic acid) \\
\hline Bioactive peptide/protein \\
\hline Complex three-dimensional \\
(3D) models \\
BIOPHYSICAL PROPERTIES \\
Stiffness
\end{tabular}
Ulrich et al. (2009), Pathak and Kumar (2012), Ruiz-Ontañon et al. (2013), Fernandez-Fuente et al. (2014), Rape and Kumar (2014), Umesh et al. (2014), Wong et al. (2015), and Grundy et al. (2016) Rao et al. (2013a), Jain et al. (2014), Kievit et al. (2014, 2016), and Cha et al. (2016) Kievit et al. (2014) and Ma et al. (2016a) Ma et al. (2012) Tamaki et al. (1997), Cordes et al. (2003), Sarkar et al. (2006), Ulrich et al. (2009), Ananthanarayanan et al. (2011), Pathak and Kumar (2012), Ruiz-Ontañon et al. (2013), Jain et al. (2014), Kim and Kumar (2014), Rape and Kumar (2014), Umesh et al. (2014), Wang et al. (2014), Rape et al. (2015), Wong et al. (2015), Heffernan et al. (2016), Ma et al. (2016a), and Ngo and Harley (2017)

Ma et al. (2012), Pathak and Kumar (2012), Rao et al. (2013a), Jain et al. (2014), Herrera-Perez et al. (2015), Pedron et al. (2015), Rape et al. (2015), Cha et al. (2016), Fan et al. (2016), Li et al. (2016), and Chonan et al. (2017)

Stiffness

Kim et al. (2008), Ulrich et al. (2009, 2010), Yang et al. (2010), Ananthanarayanan et al. (2011), Pathak and Kumar (2012), Florczyk et al. (2013, 2016), Pedron and Harley (2013), Pedron et al. (2013, 2015), Rao et al. (2013a,b), Fernandez-Fuente et al. (2014), Heffernan et al. (2014, 2016, 2017), Kim and Kumar (2014), Rape and Kumar (2014), Umesh et al. (2014), Wang et al. (2014), HerreraPerez et al. (2015), Rape et al. (2015), Wong et al. (2015), Cha et al. (2016), Grundy et al. (2016), Chen et al. (2017), and Ngo and Harley (2017)

Porosity Kim et al. (2008), Yang et al. (2010, 2014), Ananthanarayanan et al. (2011), Ma et al. (2012, 2016a), Pathak and Kumar (2012), Florczyk et al. (2013, 2016), Pedron and Harley (2013), Rao et al. (2013a,b), Kievit et al. (2014, 2016), Wang et al. (2014, 2016), Herrera-Perez et al. (2015), Cha et al. (2016), Fan et al. (2016), and Oh et al. (2016)

Microchannels Pathak and Kumar (2012), Pedron et al. (2015), Fan et al. (2016), and Chonan et al. (2017)

Fibers/alignment Kim et al. (2008), Ulrich et al. (2010), Yang et al. (2010), Rao et al. (2013a,b), Jain et al. (2014), Herrera-Perez et al. (2015), Cha et al. (2016), and Ma et al. (2016a)

\section{GBM CELL LINES}

U87

Kim et al. (2008), Jin et al. (2009), Ulrich et al. (2009), Kievit et al. (2010, 2014, 2016), Ananthanarayanan et al. (2011), Eke et al. (2012), Pedron and Harley (2013), Pedron et al. (2013, 2015), Fernandez-Fuente et al. (2014), Heffernan et al. (2014), Jain et al. (2014), Jiguet Jiglaire et al. (2014), Kim and Kumar (2014), Rape and Kumar (2014), Umesh et al. (2014), Wang et al. (2014), Fan et al. (2016), Florczyk et al. (2016), and Ngo and Harley (2017)

\begin{tabular}{ll}
\hline U373 & $\begin{array}{l}\text { Jin et al. (2009), Ulrich et al. (2009, 2010), Ananthanarayanan et al. (2011), Pathak and Kumar (2012), Kim and Kumar (2014), Rape } \\
\text { and Kumar (2014), Umesh et al. (2014), Rape et al. (2015), and Wong et al. (2015) }\end{array}$ \\
\hline U251 & Sarkar et al. (2006), Jin et al. (2009), Ulrich et al. (2009), Ma et al. (2016a), and Chen et al. (2017) \\
\hline U118 & Kievit et al. (2010, 2014), Florczyk et al. (2013), and Heffernan et al. (2014, 2016) \\
\hline U138 & Cordes et al. (2003) and Eke et al. (2012) \\
\hline A172 & Cordes et al. (2003), Eke et al. (2012), and Fernandez-Fuente et al. (2014) \\
\hline U343 & Jin et al. (2009) \\
\hline T178 & Sarkar et al. (2006) \\
\hline
\end{tabular}


TABLE $1 \mid$ Continued

\begin{tabular}{|c|c|}
\hline & Reference \\
\hline LN229 & Cordes et al. (2003) and Eke et al. (2012) \\
\hline LN18 & Cordes et al. (2003) \\
\hline SNB19 & Ulrich et al. (2009) \\
\hline M059K & Ma et al. (2012) \\
\hline Genetically modified GBM & Pedron et al. (2013, 2015), Heffernan et al. (2014), and Kim and Kumar (2014) \\
\hline Coculture & Ma et al. (2012), Ruiz-Ontañon et al. (2013), Kievit et al. (2016), Chonan et al. (2017), and Ngo and Harley (2017) \\
\hline Murine model & $\begin{array}{l}\text { Tamaki et al. (1997), Ulrich et al. (2009), Kievit et al. (2010), Yang et al. (2010), Jiguet Jiglaire et al. (2014), Hubert et al. (2016), and } \\
\text { Chonan et al. (2017) }\end{array}$ \\
\hline Patient derived & $\begin{array}{l}\text { Eke et al. (2012), Rao et al. (2013a,b), Ruiz-Ontañon et al. (2013), Fernandez-Fuente et al. (2014), Jiguet Jiglaire et al. (2014), Yang } \\
\text { et al. (2014), Herrera-Perez et al. (2015), Wong et al. (2015), Cha et al. (2016), Grundy et al. (2016), Hubert et al. (2016), Li et al. } \\
\text { (2016), Oh et al. (2016), Wang et al. (2016), Heffernan et al. (2017), and Pedron et al. (2017) }\end{array}$ \\
\hline
\end{tabular}

\section{BIOLOGICAL BEHAVIORS}

Two-dimensional migration

Kim et al. (2008), Jin et al. (2009), Ulrich et al. (2009), Ananthanarayanan et al. (2011), Eke et al. (2012), Pathak and Kumar (2012), Rao et al. (2013a), Ruiz-Ontañon et al. (2013), Fernandez-Fuente et al. (2014), Kim and Kumar (2014), Rape and Kumar (2014), Wong et al. (2015), Grundy et al. (2016), and Chonan et al. (2017)

3D invasion Tamaki et al. (1997), Cordes et al. (2003), Sarkar et al. (2006), Kim et al. (2008), Jin et al. (2009), Ulrich et al. (2010), Yang et al. (2010), Ananthanarayanan et al. (2011), Cheng et al. (2011), Eke et al. (2012), Pathak and Kumar (2012), Florczyk et al. (2013), Rao et al. (2013b), Ruiz-Ontañon et al. (2013), Heffernan et al. (2014, 2016), Jain et al. (2014), Kim and Kumar (2014), Rape and Kumar (2014), Herrera-Perez et al. (2015), Wong et al. (2015), Cha et al. (2016), Grundy et al. (2016), Chen et al. (2017), and Chonan et al. (2017)

Proliferation Tamaki et al. (1997), Ulrich et al. (2009), Kievit et al. (2010, 2014, 2016), Yang et al. (2010, 2014), Ananthanarayanan et al. (2011), Eke et al. (2012), Ma et al. (2012, 2016a), Florczyk et al. (2013, 2016), Pedron and Harley (2013), Pedron et al. (2013, 2015, 2017), Ruiz-Ontañon et al. (2013), Heffernan et al. (2014, 2016, 2017), Jain et al. (2014), Jiguet Jiglaire et al. (2014), Umesh et al. (2014), Wang et al. (2014, 2016), Wong et al. (2015), Cha et al. (2016), Hubert et al. (2016), Li et al. (2016), and Chen et al. (2017)

Malignancy markers C Cordes et al. (2003), Sarkar et al. (2006), Kim et al. (2008), Jin et al. (2009), Kievit et al. (2010, 2014, 2016), Cheng et al. (2011), Eke et al. (2012), Florczyk et al. (2013, 2016), Pedron and Harley (2013), Pedron et al. (2013, 2015, 2017), Rao et al. (2013a), Ruiz-Ontañon et al. (2013), Fernandez-Fuente et al. (2014), Jiguet Jiglaire et al. (2014), Kim and Kumar (2014), Umesh et al. (2014), Wang et al. (2014, 2016), Yang et al. (2014), Herrera-Perez et al. (2015), Rape et al. (2015), Wong et al. (2015), Cha et al. (2016), Hubert et al. (2016), Li et al. (2016), Ma et al. (2016a), Chen et al. (2017), Chonan et al. (2017), and Heffernan et al. (2017)

Stem phenotypes Cheng et al. (2011), Florczyk et al. (2013, 2016), Ruiz-Ontañon et al. (2013), Fernandez-Fuente et al. (2014), Kievit et al. (2014, 2016), Yang et al. (2014), Herrera-Perez et al. (2015), Wong et al. (2015), Cha et al. (2016), Grundy et al. (2016), Hubert et al. (2016), Li et al. (2016), Ma et al. (2016a), Wang et al. (2016), Chonan et al. (2017), Heffernan et al. (2017), and Pedron et al. (2017)

In vivo characteristics Kievit et al. (2010, 2014), Cheng et al. (2011), Ruiz-Ontañon et al. (2013), Jain et al. (2014), Jiguet Jiglaire et al. (2014), Yang et al. (2014), Wong et al. (2015), Florczyk et al. (2016), Hubert et al. (2016), Li et al. (2016), and Pedron et al. (2017)

TREATMENT RESPONSE

Chemotherapy

Kim et al. (2008), Ulrich et al. (2009), Eke et al. (2012), Ma et al. (2012), Pathak and Kumar (2012), Florczyk et al. (2013), FernandezFuente et al. (2014), Jain et al. (2014), Jiguet Jiglaire et al. (2014), Rape and Kumar (2014), Umesh et al. (2014), Yang et al. (2014), Cha et al. (2016), Fan et al. (2016), Wang et al. (2016), and Pedron et al. (2017)

Radiation Cordes et al. (2003), Eke et al. (2012), Jiguet Jiglaire et al. (2014), Hubert et al. (2016), and Heffernan et al. (2017) hydrophilic ECM proteins (Rao et al., 2013a; Cha et al., 2016; Ma et al., 2016a). Scaffolds of either classification can be further designed as porous, fibrous, anisotropic, or some combination, each with varying degrees of control of these physical properties dependent on the constituents. These properties enable modeling of a wide variety of topographies to mimic the physiological microenvironment. Chemical and physical cross-linking reactions are often necessary to increase the molecular weight of a biomaterial such that it forms an insoluble physical structure in aqueous solution. Strategies that do not negatively impact cell viability are particularly desirable. For chemically cross-linked biomaterials, click chemistry, such as Michael addition (Ananthanarayanan et al., 2011; Heffernan et al., 2014; Jiguet Jiglaire et al., 2014; Kim and Kumar, 2014; Rape and Kumar, 2014; Rape et al., 2015), describes stepwise reactions that proceed efficiently at neutral $\mathrm{pH}$, do not require biologically damaging solvents or reaction conditions, and do not produce any cytotoxic byproducts (Hoyle et al., 2010). Another common example of chemical cross-linking is UV free radical polymerization, which, unlike most free radical reactions, may utilize an aqueous compatible initiator that is photoreactive (e.g., Irgacure 2959). This method enables polymerization of reactive monomers such as terminal olefins (e.g., acrylates) 
(Pedron and Harley, 2013; Pedron et al., 2013, 2015, 2017; Chen et al., 2017; Ngo and Harley, 2017). Alternatively, physical cross-linking proceeds without a chemical reaction; alterations in $\mathrm{pH}$ or temperature produce electrostatic interactions that result in polymerization and/or precipitation (El-Sherbiny and Yacoub, 2013); typical examples include collagen (pH stimulus) or poly( $N$-isopropylacrylamide) (temperature stimulus). It is well known that the degree of cross-linking (chemical or physical) for any given material will affect the porosity, density, and stiffness of the scaffold, which are each independently important considerations in GBM tissue engineering.

\section{D Culture Methods}

Biomaterial cultures are performed with cells or spheroids either seeded on the scaffold surface or encapsulated within the scaffold during cross-linking. Surface cultures enable measurement of cellular behaviors (motility, invasion, proliferation, viability, etc.) in response to the biophysical and biochemical material properties. They also provide a set initial location for cells and do not necessarily require biodegradation of the material to allow for cell proliferation or motility, since cells are capable of moving across the surface. Encapsulation cultures offer a more physiologically relevant scenario but require biocompatible cross-linking and matrix degradation for cell growth and motility. Both scenarios are regularly used to measure invasive capacity, which cannot be fully recapitulated in $2 \mathrm{D}$ in vitro systems.

For both surface and encapsulation cultures, biological assays must be either performed in situ (e.g., immunofluorescence and cell tracking) or alternatively, on cells recovered from the scaffold (e.g., western blot, polymerase chain reaction, and fluorescence activated cell sorting). Both culture approaches pose various technical challenges to performing these assays that are specific to the biomaterial system; a significant consideration in designing a 3D culture format. For example, chemically crosslinked materials may require degradation or cell dissociation conditions that adversely affect cell viability or the presentation of surface proteins. Physical scaffolds, on the other hand, may offer reversible formation in response to mild environmental changes and thereby enable easy cell recovery for post-culture analysis.

\section{Biophysical and Biochemical Regulation of GBM Behaviors}

The in vivo tumor microenvironment provides critical regulatory functions for GBM tumors. As a result, there are many reports investigating how the physical microarchitecture and biochemical features of 3D biomaterials regulate or elicit specific GBM behaviors in vitro (summarized in detail in Table 1). These studies have been reviewed in great detail elsewhere (Rao et al., 2014; Rape et al., 2014; Xiao et al., 2017). One well-established observation is that matrix stiffness and topography (porosity, fiber content, geometry) can alter cellular phenotype to elicit malignant behaviors, including proliferation, migration, and invasion. Mechanosensation, or the ability for cells to sense mechanical forces and stiffness, is a key component of GBM biology that mediates tumor growth and cell motility (Rape et al., 2014). This was demonstrated in vitro by Ulrich et al. (2009) who described that GBM cell lines displayed both higher motility and proliferation on high stiffness substrates and also identified that the motile responses were governed at least in part by non-muscle myosin II. Furthermore, one of the more intriguing developments in understanding biophysical regulation has been the discovery that biomaterial fibers mimicking the structure of blood vessels are capable of encouraging and guiding GBM invasion (Rao et al., 2013a; Jain et al., 2014; Herrera-Perez et al., 2015; Cha et al., 2016). The primary method for changing the biophysical properties of 3D models is via altering the concentration of the constituent biomaterials. While effective, this often also coupled with changing the density of bioactive components. Isolating the impact of different biophysical cues on cellular behavior is almost impossible in vivo, but biomaterial platforms offer an opportunity to independently manipulate these variables, which will be important to deepening understanding of the disease (Wang et al., 2014).

In considering the biochemical influence of the microenvironment on GBM biology, various different scaffold components have been explored (complete list in Table 1); one of the most common materials utilized for this purpose is HA. Given that HA, discussed earlier, has many essential functions in GBM (Giese and Westphal, 1996), it is unsurprising that HA hydrogels have been shown to regulate a wide variety of behaviors including proliferation (Pedron et al., 2013), invasion (Heffernan et al., 2014), stem phenotypes (Cha et al., 2016), and treatment resistance (Jiguet Jiglaire et al., 2014). HA does not provide cellular adhesion sites, and as a result is regularly modified with cell adhesion peptides or combined with other biomaterials, such as collagen, to enable cell attachment (Ananthanarayanan et al., 2011; Pedron et al., 2013; Heffernan et al., 2014; Jiguet Jiglaire et al., 2014). While relatively insignificant components of the native brain ECM, collagen and Matrigel ${ }^{\circledR}$ are also prominent biomaterials in GBM research primarily because of their high density of focal adhesion sites, their history of use in other cancer models (e.g., breast cancer), and the ease with which they can be experimentally implemented. Both models have been used to investigate the role of matrix signaling in promoting focal adhesion mediated GBM invasion (Herrera-Perez et al., 2015; Heffernan et al., 2016). Alternatively, PEG-based biomaterials offer a tunable synthetic platform with the potential for easy chemical modification. PEG gels have been designed to be enzymatically degradable (Wang et al., 2014), hydrolytically stable or degradable (Heffernan et al., 2014), or modified with cell instructive peptides (Ananthanarayanan et al., 2011), each of which have been shown to modify malignant GBM behaviors. Moreover, complex multicomponent biomaterial models have also been reported for developing high-throughput studies of GBM behaviors across a range of different microenvironmental conditions (Pedron et al., 2015), or alternatively, through cocultures with microenvironmental support cells within a single 3D in vitro system (Ngo and Harley, 2017). Together, the current body of work (Table 1) illustrates the breadth of understanding that has developed in response to implementing in vitro biomaterial cultures. 


\section{BIOMATERIALS FOR PROBING GSC BIOLOGY}

\section{Engineering the Stem Cell Microenvironment}

Engineering GSC instructive in vitro microenvironments is a relatively new approach derived from well-established tissue engineering research. Stem cells are widely regarded for their potential to regenerate and establish functional tissues. Neural tissue engineering, which is most closely related to methods for modeling the GBM microenvironment, primarily focuses on developing novel techniques for directing NSC behaviors. In this field, biomaterials have been utilized to elucidate various stem cell behaviors with a focus on understanding how biophysical and biochemical factors in 2D and 3D environments affect NSC maintenance, self-renewal, and differentiation mechanisms (Teixeira et al., 2007). Through these studies, the mechanical stiffness of culture substrates and matrices has been identified as a potent regulator of NSC fate. Saha et al. (2008) reported that NSC differentiation could be directed with soft substrates $(100-500 \mathrm{~Pa})$ to promote neurogenesis and stiff $(>1,000 \mathrm{~Pa})$ matrices to promote gliogenesis. In addition to matrix stiffness, Soen et al. (2006) and Nakajima et al. (2007) demonstrated that specific ECM components and growth factors were also capable of controlling stem cell fate and differentiation in culture. Other cellular components of the NSC microenvironment have been investigated as regulators of stem cell fate in vitro. For example, Shen et al. (2004) determined that endothelial cells secrete soluble factors that promote and maintain stem phenotypes in NSC populations.

The regenerative capacity of NSCs has also been investigated in $3 \mathrm{D}$ microenvironment models with both matrix composition and stiffness again identified as key regulatory components. Here, Saha et al. (2008) described that very soft substrates $(<100 \mathrm{~Pa})$ promoted quiescent NSC phenotypes, while stiffening these substrates $(\geq 100 \mathrm{~Pa})$ promoted expansion of the NSC pool. The structure of the ECM is also important to NSC neural regenerative properties, as Yang et al. (2005) described scaffolds composed of aligned poly(L-lactic acid) nanofibers promoted neuronal phenotypes and neurite outgrowth along the fibers. More biomimetic approaches have also been tested using ECM components of the in vivo NSC niche as well. To this end, Cheng et al. (2013b) described that a laminin-derived IKVAV peptide-based hydrogel supported NSC neuronal differentiation and improved tissue regeneration in vivo following a traumatic brain injury. In addition, we reported that an HA-laminin composite hydrogels increase the migratory response of NSCs a result of increased sensitivity to stromal cell-derived factor $1 \alpha$ both in vitro and in vivo (Addington et al., 2015, 2017).

Similar regulatory mechanisms govern both GSC and NSC biology (Sanai et al., 2005), and as such, these examples have direct relevance to understanding and predicting how model microenvironments may affect malignant GSC phenotypes. In applying these same tissue engineering approaches to GSCs, conditions under which these cells acquire or enhance stem phenotypes, prefer to initiate invasive mechanisms, or exhibit treatment resistance have been identified. These results provide better understanding of the underlying mechanisms that drive microenvironmental support for GSC populations.

\section{GBM Stem Plasticity in 3D Culture}

Glioblastoma stem-like cells and NGSCs are believed to exist in a regulated state of plasticity where induction of differentiation is a bidirectional process regulated by the microenvironment, epigenetics, and response to treatment (Heddleston et al., 2009; Dahan et al., 2014; Safa et al., 2015). This stem plasticity has been investigated using immortalized GBM cell lines as a model of NGSCs. Although, as previously described, these cell lines do not offer a complete and accurate depiction of GBM biology, and the mechanisms that are employed to acquire stem phenotypes may mimic GSC plasticity (Jacobs et al., 2011; Zhang et al., 2013).

Stem plasticity has been studied in GBM cell lines cultured in chitosan-based scaffolds; for example, Florczyk et al. (2013) developed a chitosan-HA composite scaffold that elucidated stem-like characteristics in U118 cells. The authors reported that these scaffolds promoted sphere formation, expression of stem markers (Nestin, Musashi-1, and CD44), and increased invasive capacity compared with traditional 2D cultures. In addition, scaffold-cultured cells displayed increased resistance to both TMZ and doxorubicin, coupled with increased expression of the ABCG2 drug efflux pump, suggesting a phenotypic switch toward a more GSC-like state (Florczyk et al., 2013). In a followup study, Kievit et al. (2014) used a chitosan-alginate scaffold to also examine stem plasticity. Using these models, U118 and U87 GBM cells again displayed increased stem protein and gene expression (CD133, Nestin, CD44, Notch, among others) in scaffold environments, which was again a function of scaffold composition. Functionally, scaffold grown cells also exhibited increased tumorigenicity in a flank tumor model. Kievit et al. (2016) further optimized this approach by coating chitosan-alginate scaffolds with HA and establishing a $3 \mathrm{D}$ coculture model of U87 and endothelial cells. These conditions also increased expression of CD133, ID1, and CD44 but interestingly slowed the growth of spheroids. Outside of GBM, Florczyk et al. (2016) employed this platform to enrich CD133 expression in prostate, breast, and liver cancer cells.

Chitosan-based scaffolds are also not the only biomaterial platform that has been reported to drive stem plasticity, as $\mathrm{Ma}$ et al. (2016a) also identified stem-specific responses to 3D electrospun polystyrene scaffolds coated with a library of seven different isoforms of laminin. The resulting behavior of U251 cells was, similar to the chitosan studies, contingent both on 3D context and matrix chemistry. Specifically, 3D scaffolds presenting the laminin isoforms $411,421,511$, and 521 promoted an increase in expression of the GSC markers (including, for example, integrin $\alpha 6$, SOX2, and OLIG2) that coincided with an increase in clonogenicity of these cells (Ma et al., 2016a).

Together, these works emphasize the significance of using engineered microenvironments to drive relevant GSC behaviors in culture. The use of immortalized cell lines provides some insight into how GBM cells exhibit plasticity in a shift from differentiated phenotypes to more stem-like behaviors. 


\section{Biomaterials Promoting GSC Expansion and Enrichment}

Engineered tumor microenvironments have also been designed to assay conditions under which patient-derived GSCs may be enriched in vitro. GSCs are typically maintained in non-adherent neurosphere conditions (Singh et al., 2003) or in adherent cultures on laminin (Pollard et al., 2009), with the desired condition often selected based on cellular affinity. In general, neurosphere conditions are most common as sphere forming capacity is regularly accompanied by a broader array of GSC specific phenotypes (self-renewal, multipotency, and stem-marker expression) (Venugopal et al., 2012). However, neurosphere culture has well-characterized drawbacks. Specifically, as spheres increase in size, the constituent cells experience differential access to oxygen and soluble signaling factors as a result of diffusion limitations (Woolard and Fine, 2009). This problem is amplified by variations in rates of cell proliferation and fusion of adjacent spheres. As a result, a single neurosphere may contain a heterogeneous mixture of clonogenic, differentiated, apoptotic, and necrotic cells (Bez et al., 2003; Beier et al., 2008; Pollard et al., 2009; Woolard and Fine, 2009).

A number of biomaterials have been described as useful tools for addressing problems associated with neurosphere aggregation. For example, Yang et al. (2014) reported that gelatin foam scaffolds maintained GSC protein expression, while also increasing HIF $1 \alpha$ and VEGF signaling to provide a GSC supportive microenvironment. As stated previously (see Therapeutic Challenges and Opportunities), hypoxia signaling, including HIFs and VEGF, has been proposed as a potential mechanism for sensitizing GSCs to treatment. Thus, this platform may be relevant for testing these hypotheses. In a separate study, Oh et al. (2016) reported that GSCs encapsulated in an alginate-PEG hydrogel formed neurospheres with relative uniformity in size, which may improve nutrient and oxygen access. Li et al. (2016) used a similar approach to expand patient-derived GSCs in a temperatureresponsive PNIPAAm-based scaffold. In this context, cells were capable of high density culture without aggregating, thus overcoming a key drawback to traditional neurosphere cultures. This system subsequently enabled improved cellular yield from GSC cultures while maintaining multipotency and stem-marker expression.

Beyond GSC expansion, conditions under which GSC phenotypes are actively enriched have also been explored in 3D culture. Chitosan-HA scaffolds were recently applied to patient-derived GSCs by Wang et al. (2016) and were found to increase stem gene expression (SOX2, and TAZ, NANOG), invasion gene expression (TWIST1, TWIST2, SNAIL1, SNAIL2, and ZEB2), and expression of genes that drive drug resistance (MGMT, HIF1A, and SOD1) compared with cells cultured as a $2 \mathrm{D}$ monolayer. GSCs cultured in these scaffolds also exhibited higher tolerance to the chemotherapeutics TMZ, carmustin (BCNU), and lomustine (CCNU). Similarly, we recently reported another set of $3 \mathrm{D}$ culture conditions that promote GSC enrichment utilizing temperature-responsive PNIPAAm-co-Jeffamine (PNJ) scaffolds (Heffernan et al., 2017). This culture platform increased self-renewal capacity, expression of the stem marker Nestin, and EGFR expression while maintaining cellular multipotency in two genetically distinct models of GBM. In addition, we observed that PNJ cultured cells also exhibited increased resistance to clinical dosages of radiation following $3 \mathrm{D}$ culture. EGFR signaling has been shown to be an important mediator of medulloblastoma radioresistance, and this platform may help to elucidate whether this mechanism is applicable to GSCs (Hambardzumyan et al., 2008).

In total, these studies suggest that there are a diverse set of biomaterials capable of maintaining GSCs cultures, and a subset of these materials are useful for actively enriching GSC specific phenotypes. Considering the differences in scaffold composition, it is also likely that GSCs are regulated via distinct mechanisms in the described culture systems.

\section{In Vitro Models of GSC Invasion}

Invasion of neoplastic cells into healthy brain tissue has and continues to be considered the most clinically significant issue inhibiting effective GBM treatment (Berens and Giese, 1999). Considering the role, GSCs play in tumor recurrence and invasion, understanding how these cells respond to specific microenvironmental cues to promote invasive behaviors is of particular importance. In the seminal work of Cheng et al. (2011), GSCs were determined to exhibit a heightened propensity for invasion. This characteristic was first identified in vitro, using a 3D Matrigeltranswell invasion assay, and was subsequently confirmed in vivo when compared with NGSCs from a matched tumor sample (Cheng et al., 2011). This description provided a foundation for employing in vitro microenvironments to determine how the biochemical, biophysical, and cellular components of the tumor microenvironment affect GSC invasion.

Biochemical input signals from the tumor microenvironment ECM influence GSC propensity for invasion, and this hypothesis has been supported in various different in vitro paradigms. Using a library of Matrigel, collagen, and HA-collagen matrices, Herrera-Perez et al. (2015) determined that modes of GSC invasion were directly dependent on ECM chemistry. These matrices accurately modeled the stiffness of healthy brain tissue, and different preparations of collagen were used to separate the effects of matrix stiffness and collagen concentration. As a result, this study identified an interplay between matrix stiffness and chemistry that influenced invasion distance and velocity. Interestingly, soluble HA (non-immobilized) decreased GSC invasion in HAcollagen matrices, and Matrigel coated microfibers, mimicking the structure of blood vessels, encouraged directional strand motility reminiscent of white matter tract invasion tendencies in vivo (Herrera-Perez et al., 2015).

Identification of biochemical pathways that promote or inhibit GSC invasion is necessary for complete characterization of these behaviors but is often underreported; a limitation of many $3 \mathrm{D}$ culture studies. As an example, Cha et al. (2016) explored a similar paradigm to Herrera-Perez et al. by measuring GSC invasion through collagen matrices that included soluble HA and PCL fibers to model blood vessels. Yet, in apparent contrast to the prior study, Cha et al. (2016) reported that soluble HA increased GSC invasion in collagen matrices, while also exhibiting increased expression of CD44, and HA synthase. In addition, treatment with an HA synthase inhibitor decreased invasion and 
effected an increase in FAK and MMP2 expression (Cha et al., 2016). While the functional results of these studies (i.e., invasion) appear contradictory, it is important to recognize differences in methodology which include the source of patient-derived cells, and the concentrations of collagen and HA in the model systems. Therefore, molecular level descriptions may improve cross-study comparisons and allow for more robust descriptions of GSC invasive mechanisms.

From analysis of the in vivo tumor microenvironment, it is clear that non-GBM cells, such as endothelial cells, are capable of regulating GSC phenotypes and promoting invasion. This behavior was studied by Chonan et al. (2017) in which a 3D collagen gel was applied to separate a murine GSC line from endothelial cells in an engineered microfluidic invasion model. Here, endothelial cells stimulated increased invasion of Nestin expressing cells through the 3D microenvironment. These GSCs also exhibited increased expression of integrin $\alpha 2$ and $\beta 3$ in response to coculture, suggesting a potential mechanistic role for endothelial cells in promoting motility of GSCs in this model. Meanwhile, cells expressing the neuronal differentiation marker tubulin $\beta 3$ were less invasive, which agrees with prior reports of increased GSC invasive capacity vs. NGSCs (Cheng et al., 2011).

As previously stated, microenvironmental stiffness regulates the invasive capacity of GBM cell lines via mechanosensation mechanisms (Ulrich et al., 2009; Ananthanarayanan et al., 2011; Pedron and Harley, 2013; Heffernan et al., 2014; Kim and Kumar, 2014). While the stiffness of healthy brain is generally characterized between 100 and 1,000 Pa, GBM tumors can present significantly increased stiffness due to their high cellularity and dense ECM (Netti et al., 2000; Georges et al., 2006; Saha et al., 2008; Buxboim et al., 2010). At present, reports investigating the effects of matrix stiffness on GSC motility in both 2D and 3D paradigms describe a complex relationship (Ruiz-Ontañon et al., 2013; Herrera-Perez et al., 2015; Wong et al., 2015; Grundy et al., 2016). Ruiz-Ontañon et al. (2013) reported that GSCs harvested from different tumor regions (peritumoral vs. bulk tumor) display invasive tendencies and sensitivity to microenvironmental stiffness that was a function of their regional origin. Unsurprisingly, peritumoral GSCs were observed to have a heightened invasive capacity. These behaviors were modeled on 2D laminin functionalized polyacrylamide matrices, within 3D Matrigel and collagen I hydrogels, as well as in chicken embryo and mouse xenografts. Moreover, peritumoral invasion was insensitive to stiffness as a result of Rac and RhoA signaling activation, and integrin $\alpha \mathrm{V} \beta 3$, an RGD peptide binding integrin, was identified as a key regulator of GSC invasion and potential target for therapy (Ruiz-Ontañon et al., 2013). Similarly, Wong et al. (2015) also reported that GSCs exhibited an insensitivity to matrix stiffness on 2D laminin coated polyacrylamide matrices. Here, matrix stiffness ranging from $80 \mathrm{~Pa}$ to $119 \mathrm{kPa}$ produced no effect on cellular migration. However, in contrast to the previous study, activation of myosin II signaling via genetic constitutive activation of RhoA, ROCK, or MLCK sensitized cells to matrix stiffness and effected a decrease in motility on soft matrices.

Together, these studies suggest that GSCs employ diverse invasion strategies that may be cell-type specific. This hypothesis was supported by Grundy et al. (2016) who suggested that a GSC subtype-specific relationship exists between invasive behavior and sensitivity to microenvironmental stiffness. In this study, migration and invasion were measured on 2D Matrigel coated polyacrylamide matrices with varying stiffness $(200 \mathrm{~Pa}-50 \mathrm{kPa})$ and also within soft $(\sim 400 \mathrm{~Pa}) 3 \mathrm{D}$ Matrigel hydrogels. With this platform, the invasive behavior of neural subtype GSCs was observed to be insensitive to stiffness, while mesenchymal subtype GSCs exhibited stiffness dependent motility. The authors surmise that the cell of origin (neural GSCs-neuronal lineage; mesenchymal GSCs-astrocytic lineage) may be a primary factor influencing GSC motility in response to microenvironmental stiffness (Grundy et al., 2016). This hypothesis also draws relevance back to the NSC paradigm, in which neuronal phenotypes manifest on soft matrices while astrocytic phenotypes dominate on stiff substrates (Saha et al., 2008).

These $3 \mathrm{D}$ invasion studies provide unique opportunities to isolate specific microenvironmental features (chemistry, stiffness, architecture, cellular support, etc.) and may be instrumental in identifying targets for therapy to address GSC invasion at the clinical level. However, the wide range of reported results indicate that a more comprehensive picture of subtype-specific and context-specific molecular mechanisms of invasion may be necessary to develop predictive hypotheses.

\section{Modeling Treatment Resistance and the Influence of Tumor Heterogeneity}

Tumors generated through orthotopic transplant of human GSCs display treatment resistance that is supported by the tumor microenvironment (Mannino and Chalmers, 2011). Yet, similar to challenges faced in studying GBM invasion, direct identification of specific resistance promoting factors remains challenging in vivo; the mechanisms underlying microenvironmental contributions to treatment resistance can be efficiently modeled in vitro. For example, Fernandez-Fuente et al. (2014) proposed that resistance to sunitinib induced receptor tyrosine kinase (RTK) inhibition is mediated by interactions specific to a $3 \mathrm{D}$ microenvironment. Using a number of different GSC, NGSC, and established GBM cell lines, the authors determined that GSCs were comparatively insensitive to RTK inhibition in $3 \mathrm{D}$ collagen gels vs. standard $2 \mathrm{D}$ conditions and $2 \mathrm{D}$ collagen coated polyacrylamide. The observed resistance was abrogated via chemical inhibition of the PI3K/Akt and MEK/ERK signaling pathways leading the authors to hypothesize that focal adhesions in $3 \mathrm{D}$ were responsible for promoting RTK resistance. Notably, changes in collagen content, stiffness (2D and $3 \mathrm{D}$ ), and soluble $\mathrm{HA}$ inclusion in $3 \mathrm{D}$ collagen gels did not produce a measurable effect on drug sensitivity.

The biochemical response to matrix bound HA has also been identified as a regulator of GSC resistance to chemotherapy in 3D culture. In a recent study by Pedron et al. (2017), the EGFR inhibitor erlotinib produced little GSC cytotoxicity in gelatin hydrogels, and its effects were predictably dependent on basal EGFR status $\left(\mathrm{EGFR}^{\mathrm{wt}}, \mathrm{EGFR}^{+}\right.$, and the GBM specific constitutively active form $\mathrm{EGFR}^{\mathrm{VIII}}$ ). In addition, incorporation of HA within the gelatin hydrogels increased erlotinib resistance in EGFR ${ }^{\mathrm{VIII}}$ cells, while 
inhibition of EGFR and CD44 increased cytotoxic effects in $\mathrm{EGFR}^{\mathrm{wt}}$ and $\mathrm{EGFR}^{+}$cells. This study provides evidence for EGFRCD44 signaling interactions that promote GSC resistance to RTK inhibition dependent on the microenvironment and molecular profile of the GBM cells. Considering the clinical importance of EGFR in GBM, this mechanism may be highly relevant designing novel inhibition strategies for GSCs. Moreover, measuring divergent responses as a function of EGFR signaling provides an example of how tumor heterogeneity may negatively impact treatment.

The development of tumor heterogeneity diminishes sensitivity to treatment as a result of divergent phenotypes (proliferative vs. quiescent, invasive vs. stationary, protein expression, etc.). Hubert et al. (2016) modeled this process by culturing GSCs in Matrigel coupled with continuous mechanical agitation. This model generated large GBM organoids with hypoxic cores that were composed of populations of GSCs and NGSCs. GSCs were primarily located at the organoid rim but were also sporadically identified in regions of hypoxia. Moreover, the GSC populations within the organoids displayed resistance to apoptosis following radiation treatment, while NGSCs were observed to be sensitive to treatment. This test demonstrates a prevailing GSC theory that conventional modes of treatment may effectively target NGSCs but leave GSCs relatively unharmed. Finally, organoid cultures were orthotopically implanted and formed tumor architecture and single-cell invasive patterns that were a better representation of the parent tumor than matched cells in neurosphere culture (Hubert et al., 2016). Thus, developing models that can recapitulate tumor heterogeneity may provide avenues for determining patient-specific drug responses via personalized medicine.

\section{CRITICAL PERSPECTIVE AND FUTURE DIRECTIONS}

Biomaterial models of the GBM tumor microenvironment have been useful to interrogate aspects of GSC biology that could not be studied easily under standard 2D culture conditions or with in vivo tumor models. While this has undoubtedly leads to progress in GBM research, there remain opportunities for improving the overall impact of these studies. Primarily, the choice of cell line remains one of the most important variables to the biological relevance of model microenvironments. Immortalized cell lines simply do not provide accurate representation of the disease in this area and should be restricted to proof of concept use if possible. Ultimately, biomaterial models are designed to identify biological features that are important to the in vivo scenario. Therefore, low-passage patient-derived cell sources that have been validated to retain genotypic and phenotypic features of the parent tumor, such as GSCs, should be prioritized. The impact of these studies will be further increased by providing detailed characterization data on both the cell lines and biomaterial system employed. Incomplete descriptions often omit key information needed to replicate studies or draw broader conclusions about GBM behaviors. One important consideration in utilizing patient-derived cells is that the heterogeneity of GBM tumors makes it unlikely that cells from different sources will behave identically. However, understanding these differences will be essential to making progress in the treatment of human disease. By classifying GSC lines into the clinically accepted subtypes, providing gene and/or protein expression data, and reporting comprehensive behavioral analyses, the field can gain a more comprehensive understanding intra- and interpatient heterogeneity. We further propose that the definition of a GSC should be considered carefully and well defined for the purpose of each study: cell behaviors should be characterized in more than one context, and standard characterization should include features such as self-renewal capacity, expression of stem related genes or proteins, their ability to differentiate into multiple lineages, and the behavior of cells transplanted in vivo. Similarly, microenvironmental variables should be tightly controlled, with consideration given to the potential interdependence of different scaffold properties. Given the role that both biochemical and biophysical signaling play in GSC regulation, decoupling these responses concepts may be key to truly isolating these biological relationships.

Looking forward, modeling GSC behaviors in engineered microenvironments provides significant opportunities for the advancement of GBM research and eventual translation of new therapies to target this population. The primary motivator is to identify treatable mechanisms or biochemical pathways that are critical for GSC persistence, invasion, or tumorigenesis. To this end, efforts focused developing heterogeneity via microenvironmental cues are particularly impactful, as heterogeneity limits the capacity for non-personalized therapies to be successful. However, if heterogeneity in recurrent tumors develops as a result of interactions with the microenvironment, there may be models that could identify key mechanisms in this process that would enable prevention. Alternatively, models designed to enable long-term maintenance of parental tumor features would also be a welcome innovation. Following resection, patientderived cell line models lose their heterogeneity, converge on a dominant phenotype, and experience genetic drift over time. Thus, models that are able to maintain and enrich GSCs with minimal plastic culture, or even straight from the patient, would be enable analysis on samples that represent the original tumor. Microenvironmental modeling may also better our understanding of the role of GSCs in driving angiogenesis. We understand that GSCs respond to hypoxic microenvironments by recruiting endothelial cells, and in some cases differentiating into vascular support cells to vascularize the tumor. However, these behaviors have not been fully demonstrated in vitro. Employing a hypoxic microenvironment model in combination with cocultured endothelial cells and microenvironmental components designed to encourage vessel formation (laminin proteins, VEGF, etc.) may enable elucidation of these processes and their mechanisms. Finally, personalized medicine options remain a long-standing goal of tumor microenvironment models. Considering again the heterogeneity of GBM, platforms that enable high-throughput testing of patient-derived tumor samples could allow for therapies to be tailored for different individuals. Such models could focus on treating tumorigenesis, invasion, or GSC maintenance. 


\section{CONCLUSION}

Although GBM is aggressively treated, conventional radiation and chemotherapy are relatively ineffective, and tumor recurrence is nearly inevitable. The GBM microenvironment is broadly protective to various tumor and tumor-associated cells; specialized niches therein provide critical functions for maintaining a population of treatment resistant GSCs that fuel tumor recurrence. Importantly, a growing body of evidence suggests that these microenvironments directly support treatment resistance and induction of stem plasticity through a diverse set of dynamic interactions. In vitro studies utilizing 3D scaffolds are proven tools for identifying and isolating microenvironmental mechanisms that regulate GBM and GSC behaviors. Therefore, we propose that further development of these models may facilitate better

\section{REFERENCES}

Addington, C. P., Dharmawaj, S., Heffernan, J. M., Sirianni, R. W., and Stabenfeldt, S. E. (2017). Hyaluronic acid-laminin hydrogels increase neural stem cell transplant retention and migratory response to SDF-1 $\alpha$. Matrix Biol. 60-61, 206-216. doi:10.1016/j.matbio.2016.09.007

Addington, C. P., Heffernan, J. M., Millar-Haskell, C. S., Tucker, E. W., Sirianni, R. W., and Stabenfeldt, S. E. (2015). Enhancing neural stem cell response to SDF-1 $\alpha$ gradients through hyaluronic acid-laminin hydrogels. Biomaterials 72, 11-19. doi:10.1016/j.biomaterials.2015.08.041

Akiyama, Y., Jung, S., Salhia, B., Lee, S., Hubbard, S., Taylor, M., et al. (2001). Hyaluronate receptors mediating glioma cell migration and proliferation. J. Neurooncol. 53, 115-127. doi:10.1023/A:1012297132047

Allen, M., Bjerke, M., Edlund, H., Nelander, S., and Westermark, B. (2016). Origin of the U87MG glioma cell line: good news and bad news. Sci. Transl. Med. 8, 354re3. doi:10.1126/scitranslmed.aaf6853

Ananthanarayanan, B., Kim, Y., and Kumar, S. (2011). Elucidating the mechanobiology of malignant brain tumors using a brain matrix-mimetic hyaluronic acid hydrogel platform. Biomaterials 32, 7913-7923. doi:10.1016/j.biomaterials. 2011.07.005

Ariza, A., López, D., Mate, J. L., Isamat, M., Musulen, E., Pujol, M., et al. (1995). Role of CD44 in the invasiveness of glioblastoma multiforme and the noninvasiveness of meningioma: an immunohistochemistry study. Hum. Pathol. 26, 1144-1147. doi:10.1016/0046-8177(95)90278-3

Bao, S., Wu, Q., McLendon, R. E., Hao, Y., Shi, Q., Hjelmeland, A. B., et al. (2006a). Glioma stem cells promote radioresistance by preferential activation of the DNA damage response. Nature 444, 756-760. doi:10.1038/nature05236

Bao, S., Wu, Q., Sathornsumetee, S., Hao, Y., Li, Z., Hjelmeland, A. B., et al. (2006b). Stem cell-like glioma cells promote tumor angiogenesis through vascular endothelial growth factor. Cancer Res. 66, 7843-7848. doi:10.1158/0008-5472. CAN-06-1010

Bar, E. E., Lin, A., Mahairaki, V., Matsui, W., and Eberhart, C. G. (2010). Hypoxia increases the expression of stem-cell markers and promotes clonogenicity in glioblastoma neurospheres. Am. J. Pathol. 177, 1491-1502. doi:10.2353/ ajpath.2010.091021

Beier, D., Wischhusen, J., Dietmaier, W., Hau, P., Proescholdt, M., Brawanski, A., et al. (2008). CD133 expression and cancer stem cells predict prognosis in high-grade oligodendroglial tumors. Brain Pathol. 18, 370-377. doi:10.1111/ j.1750-3639.2008.00130.x

Bellail, A. C., Hunter, S. B., Brat, D. J., Tan, C., and Van Meir, E. G. (2004). Microregional extracellular matrix heterogeneity in brain modulates glioma cell invasion. Int. J. Biochem. Cell Biol. 36, 1046-1069. doi:10.1016/j. biocel.2004.01.013

Berens, M. E., and Giese, A. (1999). “...those left behind.” Biology and oncology of invasive glioma cells. Neoplasia 1, 208-219. doi:10.1038/sj.neo.7900034

Bertout, J. A., Majmundar, A. J., Gordan, J. D., Lam, J. C., Ditsworth, D., Keith, B., et al. (2009). HIF $2 \alpha$ inhibition promotes p53 pathway activity, tumor cell death, and radiation responses. Proc. Natl. Acad. Sci. U.S.A. 106, 14391-14396. doi:10.1073/pnas.0907357106 understanding of the mechanisms that maintain GSCs in the microenvironment and may precede the development of new methods for disrupting niche regulation.

\section{AUTHOR CONTRIBUTIONS}

JMH and RWS were equally involved in conceptualizing, writing, and editing this manuscript.

\section{FUNDING}

The authors gratefully acknowledge funding support for this work provided by the Rick Oehme Foundation and the Barrow Neurological Foundation.

Bez, A., Corsini, E., Curti, D., Biggiogera, M., Colombo, A., Nicosia, R. F., et al (2003). Neurosphere and neurosphere-forming cells: morphological and ultrastructural characterization. Brain Res. 993, 18-29. doi:10.1016/j.brainres. 2003.08.061

Bhatt, R. S., Landis, D. M., Zimmer, M., Torregrossa, J., Chen, S., Sukhatme, V. P., et al. (2008). Hypoxia-inducible factor-2 $\alpha$ : effect on radiation sensitivity and differential regulation by an mTOR inhibitor. BJU Int. 102, 358-363. doi:10.1111/j.1464-410X.2008.07558.x

Bleau, A.-M., Hambardzumyan, D., Ozawa, T., Fomchenko, E. I., Huse, J. T., Brennan, C. W., et al. (2009). PTEN/PI3K/Akt pathway regulates the side population phenotype and ABCG2 activity in glioma tumor stem-like cells. Cell Stem Cell 4, 226-235. doi:10.1016/j.stem.2009.01.007

Bonavia, R., Inda, M.-M., Cavenee, W. K., and Furnari, F. B. (2011). Heterogeneity maintenance in glioblastoma: a social network. Cancer Res. 71, 4055-4060. doi:10.1158/0008-5472.CAN-11-0153

Buxboim, A., Rajagopal, K., Brown, A. E. X., and Discher, D. E. (2010). How deeply cells feel: methods for thin gels. J. Phys. Condens. Matter 22, 194116. doi:10.1088/0953-8984/22/19/194116

Calabrese, C., Poppleton, H., Kocak, M., Hogg, T. L., Fuller, C., Hamner, B., et al. (2007). A perivascular niche for brain tumor stem cells. Cancer Cell 11, 69-82. doi:10.1016/j.ccr.2006.11.020

Cha, J., Kang, S.-G., and Kim, P. (2016). Strategies of mesenchymal invasion of patient-derived brain tumors: microenvironmental adaptation. Sci. Rep. 6, 24912. doi: $10.1038 /$ srep 24912

Charles, N., Ozawa, T., Squatrito, M., Bleau, A.-M., Brennan, C. W., Hambardzumyan, D., et al. (2010). Perivascular nitric oxide activates notch signaling and promotes stem-like character in PDGF-induced glioma cells. Cell Stem Cell 6, 141-152. doi:10.1016/j.stem.2010.01.001

Charles, N. A., Holland, E. C., Gilbertson, R., Glass, R., and Kettenmann, H. (2011). The brain tumor microenvironment. Glia 59, 1169-1180. doi:10.1002/ glia.21136

Chen, J., Li, Y., Yu, T.-S., McKay, R. M., Burns, D. K., Kernie, S. G., et al. (2012). A restricted cell population propagates glioblastoma growth after chemotherapy. Nature 488, 522-526. doi:10.1038/nature11287

Chen, J.-W. E., Pedron, S., and Harley, B. A. C. (2017). The combined influence of hydrogel stiffness and matrix-bound hyaluronic acid content on glioblastoma invasion. Macromol. Biosci. 17. doi:10.1002/mabi.201700018

Chen, Z., Htay, A., Santos, W. D., Gillies, G. T., Fillmore, H. L., Sholley, M. M., et al. (2009). In vitro angiogenesis by human umbilical vein endothelial cells (HUVEC) induced by three-dimensional co-culture with glioblastoma cells. J. Neurooncol. 92, 121-128. doi:10.1007/s11060-008-9742-y

Cheng, L., Huang, Z., Zhou, W., Wu, Q., Donnola, S., Liu, J. K., et al. (2013a). Glioblastoma stem cells generate vascular pericytes to support vessel function and tumor growth. Cell 153, 139-152. doi:10.1016/j.cell.2013. 02.021

Cheng, T.-Y., Chen, M.-H., Chang, W.-H., Huang, M.-Y., and Wang, T.-W. (2013b). Neural stem cells encapsulated in a functionalized self-assembling peptide hydrogel for brain tissue engineering. Biomaterials 34, 2005-2016. doi:10.1016/j.biomaterials.2012.11.043 
Cheng, L., Wu, Q., Guryanova, O. A., Huang, Z., Huang, Q., Rich, J. N., et al. (2011). Elevated invasive potential of glioblastoma stem cells. Biochem. Biophys. Res. Commun. 406, 643-648. doi:10.1016/j.bbrc.2011.02.123

Chonan, Y., Taki, S., Sampetrean, O., Saya, H., and Sudo, R. (2017). Endotheliuminduced three-dimensional invasion of heterogeneous glioma initiating cells in a microfluidic coculture platform. Integr. Biol. 9, 762-773. doi:10.1039/ C7IB00091J

Cordes, N., Hansmeier, B., Beinke, C., Meineke, V., and van Beuningen, D. (2003). Irradiation differentially affects substratum-dependent survival, adhesion, and invasion of glioblastoma cell lines. Br. J. Cancer 89, 2122-2132. doi:10.1038/ sj.bjc. 6601429

Dahan, P., Martinez Gala, J., Delmas, C., Monferran, S., Malric, L., Zentkowski, D., et al. (2014). Ionizing radiations sustain glioblastoma cell dedifferentiation to a stem-like phenotype through survivin: possible involvement in radioresistance. Cell Death Dis. 5, e1543. doi:10.1038/cddis.2014.509

Del Duca, D., Werbowetski, T., and Del Maestro, R. F. (2004). Spheroid preparation from hanging drops: characterization of a model of brain tumor invasion. J. Neurooncol. 67, 295-303. doi:10.1023/B:NEON.0000024220.07063.70

Deleyrolle, L. P., Harding, A., Cato, K., Siebzehnrubl, F. A., Rahman, M., Azari, H., et al. (2011). Evidence for label-retaining tumour-initiating cells in human glioblastoma. Brain 134, 1331-1343. doi:10.1093/brain/awr081

Delpech, B., Maingonnat, C., Girard, N., Chauzy, C., Olivier, A., Maunoury, R., et al. (1993). Hyaluronan and hyaluronectin in the extracellular matrix of human brain tumour stroma. Eur. J. Cancer 29, 1012-1017. doi:10.1016/ S0959-8049(05)80214-X

Eke, I., Storch, K., Kästner, I., Vehlow, A., Faethe, C., Mueller-Klieser, W., et al. (2012). Three-dimensional invasion of human glioblastoma cells remains unchanged by $\mathrm{x}$-ray and carbon ion irradiation in vitro. Int. J. Radiat. Oncol. 84, e515-e523. doi:10.1016/j.ijrobp.2012.06.012

El-Sherbiny, I. M., and Yacoub, M. H. (2013). Hydrogel scaffolds for tissue engineering: progress and challenges. Glob. Cardiol. Sci. Pract. 2013, 316-342. doi: $10.5339 /$ gcsp. 2013.38

Fael Al-Mayhani, T. M., Ball, S. L. R., Zhao, J.-W., Fawcett, J., Ichimura, K., Collins, P. V., et al. (2009). An efficient method for derivation and propagation of glioblastoma cell lines that conserves the molecular profile of their original tumours. J. Neurosci. Methods 176, 192-199. doi:10.1016/j. jneumeth.2008.07.022

Fan, Y., Nguyen, D. T., Akay, Y., Xu, F., and Akay, M. (2016). Engineering a brain cancer chip for high-throughput drug screening. Sci. Rep. 6, 25062. doi:10.1038/ srep25062

Fernandez-Fuente, G., Mollinedo, P., Grande, L., Vazquez-Barquero, A., and Fernandez-Luna, J. L. (2014). Culture dimensionality influences the resistance of glioblastoma stem-like cells to multikinase inhibitors. Mol. Cancer Ther. 13, 1664-1672. doi:10.1158/1535-7163.MCT-13-0854

Florczyk, S. J., Kievit, F. M., Wang, K., Erickson, A. E., Ellenbogen, R. G., and Zhang, M. (2016). 3D porous chitosan-alginate scaffolds promote proliferation and enrichment of cancer stem-like cells. J. Mater. Chem. B. 4, 6326-6334. doi:10.1039/C6TB01713D

Florczyk, S. J., Wang, K., Jana, S., Wood, D. L., Sytsma, S. K., Sham, J. G., et al. (2013). Porous chitosan-hyaluronic acid scaffolds as a mimic of glioblastoma microenvironment ECM. Biomaterials 34, 10143-10150. doi:10.1016/j.biomaterials. 2013.09.034

Folkins, C., Shaked, Y., Man, S., Tang, T., Lee, C. R., Zhu, Z., et al. (2009). Glioma tumor stem-like cells promote tumor angiogenesis and vasculogenesis via vascular endothelial growth factor and stromal-derived factor 1. Cancer Res. 69, 7243-7251. doi:10.1158/0008-5472.CAN-09-0167

Galan-Moya, E. M., Le Guelte, A., Lima Fernandes, E., Thirant, C., Dwyer, J., Bidere, N., et al. (2011). Secreted factors from brain endothelial cells maintain glioblastoma stem-like cell expansion through the mTOR pathway. EMBO Rep. 12, 470-476. doi:10.1038/embor.2011.39

Galli, R., Binda, E., Orfanelli, U., Cipelletti, B., Gritti, A., Vitis, S. D., et al. (2004). Isolation and characterization of tumorigenic, stem-like neural precursors from human glioblastoma. Cancer Res. 64, 7011-7021. doi:10.1158/0008-5472. CAN-04-1364

Georges, P. C., Miller, W. J., Meaney, D. F., Sawyer, E. S., and Janmey, P. A. (2006). Matrices with compliance comparable to that of brain tissue select neuronal over glial growth in mixed cortical cultures. Biophys. J. 90, 3012-3018. doi:10.1529/ biophysj.105.073114
Giese, A., Berens, M. E., and Westphal, M. (2003). Cost of migration: invasion of malignant gliomas and implications for treatment. J. Clin. Oncol. 21, 1624-1636. doi:10.1200/JCO.2003.05.063

Giese, A., Loo, M. A., Rief, M. D., Tran, N., and Berens, M. E. (1995). Substrates for astrocytoma invasion. Neurosurgery 37, 294-301. doi:10.1227/00006123199508000-00015

Giese, A., and Westphal, M. (1996). Glioma invasion in the central nervous system. Neurosurgery 39, 235-252. doi:10.1097/00006123-199608000-00001

Gilbertson, R. J., and Rich, J. N. (2007). Making a tumour's bed: glioblastoma stem cells and the vascular niche. Nat. Rev. Cancer 7, 733-736. doi:10.1038/ nrc2246

Grundy, T. J., De Leon, E., Griffin, K. R., Stringer, B. W., Day, B. W., Fabry, B., et al. (2016). Differential response of patient-derived primary glioblastoma cells to environmental stiffness. Sci. Rep. 6, 23353. doi:10.1038/srep23353

Haas, T. L., Sciuto, M. R., Brunetto, L., Valvo, C., Signore, M., Fiori, M. E., et al. (2017). Integrin $\alpha 7$ is a functional marker and potential therapeutic target in glioblastoma. Cell Stem Cell 21, 35-50.e9. doi:10.1016/j.stem.2017. 04.009

Hambardzumyan, D., Becher, O. J., Rosenblum, M. K., Pandolfi, P. P., ManovaTodorova, K., and Holland, E. C. (2008). PI3K pathway regulates survival of cancer stem cells residing in the perivascular niche following radiation in medulloblastoma in vivo. Genes Dev. 22, 436-448. doi:10.1101/gad.1627008

Harrison, L., and Blackwell, K. (2004). Hypoxia and anemia: factors in decreased sensitivity to radiation therapy and chemotherapy? Oncologist 9, 31-40. doi:10.1634/theoncologist.9-90005-31

Heddleston, J. M., Li, Z., Lathia, J. D., Bao, S., Hjelmeland, A. B., and Rich, J. N. (2010). Hypoxia inducible factors in cancer stem cells. Br. J. Cancer 102 , 789-795. doi:10.1038/sj.bjc.6605551

Heddleston, J. M., Li, Z., McLendon, R. E., Hjelmeland, A. B., and Rich, J. N. (2009). The hypoxic microenvironment maintains glioblastoma stem cells and promotes reprogramming towards a cancer stem cell phenotype. Cell Cycle 8, 3274-3284. doi:10.4161/cc.8.20.9701

Heffernan, J., Overstreet, D., Le, L., Vernon, B., and Sirianni, R. (2014). Bioengineered scaffolds for 3D analysis of glioblastoma proliferation and invasion. Ann. Biomed. Eng. 43, 1965-1977. doi:10.1007/s10439-014-1223-1

Heffernan, J. M., McNamara, J. B., Borwege, S., Vernon, B. L., Sanai, N., Mehta, S., et al. (2017). PNIPAAm-co-Jeffamine ${ }^{\circledR}$ (PNJ) scaffolds as in vitro models for niche enrichment of glioblastoma stem-like cells. Biomaterials 143, 149-158. doi:10.1016/j.biomaterials.2017.05.007

Heffernan, J. M., Overstreet, D. J., Srinivasan, S., Le, L. D., Vernon, B. L., and Sirianni, R. W. (2016). Temperature responsive hydrogels enable transient three-dimensional tumor cultures via rapid cell recovery. J. Biomed. Mater. Res. A. 104, 17-25. doi:10.1002/jbm.a.35534

Herrera-Perez, M., Voytik-Harbin, S. L., and Rickus, J. L. (2015). Extracellular matrix properties regulate the migratory response of glioblastoma stem cells in three-dimensional culture. Tissue Eng. Part A 21, 2572-2582. doi:10.1089/ ten.tea.2014.0504

Hjelmeland, A. B., Wu, Q., Heddleston, J. M., Choudhary, G. S., MacSwords, J., Lathia, J. D., et al. (2011). Acidic stress promotes a glioma stem cell phenotype. Cell Death Differ. 18, 829-840. doi:10.1038/cdd.2010.150

Holmquist-Mengelbier, L., Fredlund, E., Löfstedt, T., Noguera, R., Navarro, S., Nilsson, H., et al. (2006). Recruitment of HIF- $1 \alpha$ and HIF- $2 \alpha$ to common target genes is differentially regulated in neuroblastoma: HIF- $2 \alpha$ promotes an aggressive phenotype. Cancer Cell 10, 413-423. doi:10.1016/j.ccr.2006. 08.026

Hovinga, K. E., Shimizu, F., Wang, R., Panagiotakos, G., Van Der Heijden, M., Moayedpardazi, H., et al. (2010). Inhibition of notch signaling in glioblastoma targets cancer stem cells via an endothelial cell intermediate. Stem Cells 28, 1019-1029. doi:10.1002/stem.429

Hoyle, C. E., Lowe, A. B., and Bowman, C. N. (2010). Thiolclick chemistry: a multifaceted toolbox for small molecule and polymer synthesis. Chem. Soc. Rev. 39, 1355-1387. doi:10.1039/B901979K

Huang, P., Rani, M. R. S., Ahluwalia, M. S., Bae, E., Prayson, R. A., Weil, R. J., et al. (2012). Endothelial expression of TNF receptor-1 generates a proapoptotic signal inhibited by integrin $\alpha 6 \beta 1$ in glioblastoma. Cancer Res. 72, 1428-1437. doi:10.1158/0008-5472.CAN-11-2621

Hubbell, J. A. (1995). Biomaterials in tissue engineering. Nat. Biotech. 13, 565-576. doi: $10.1038 / \mathrm{nbt} 0695-565$ 
Hubert, C. G., Rivera, M., Spangler, L. C., Wu, Q., Mack, S. C., Prager, B. C., et al. (2016). Culture system derived from human glioblastomas recapitulates the hypoxic gradients and cancer stem cell heterogeneity of tumors found in vivo. Cancer Res. 76, 2465-2477. doi:10.1158/0008-5472.CAN-15-2402

Huszthy, P. C., Daphu, I., Niclou, S. P., Stieber, D., Nigro, J. M., Sakariassen, P. O., et al. (2012). In vivo models of primary brain tumors: pitfalls and perspectives. Neuro Oncol. 14, 979-993. doi:10.1093/neuonc/nos135

Ignatova, T. N., Kukekov, V. G., Laywell, E. D., Suslov, O. N., Vrionis, F. D., and Steindler, D. A. (2002). Human cortical glial tumors contain neural stem-like cells expressing astroglial and neuronal markers in vitro. Glia 39, 193-206. doi:10.1002/glia.10094

Ishiwata, T., Teduka, K., Yamamoto, T., Kawahara, K., Matsuda, Y., and Naito, Z. (2011). Neuroepithelial stem cell marker nestin regulates the migration, invasion and growth of human gliomas. Oncol. Rep. 26, 91-99. doi:10.3892/or. 2011.1267

Jacobs, V. L., Valdes, P. A., Hickey, W. F., and De Leo, J. A. (2011). Current review of in vivo GBM rodent models: emphasis on the CNS-1 tumour model. ASN Neuro 3, 171-181. doi:10.1042/AN20110014

Jain, A., Betancur, M., Patel, G. D., Valmikinathan, C. M., Mukhatyar, V. J., Vakharia, A., et al. (2014). Guiding intracortical brain tumour cells to an extracortical cytotoxic hydrogel using aligned polymeric nanofibres. Nat. Mater. 13, 308-316. doi:10.1038/nmat3878

Jamal, M., Rath, B. H., Tsang, P. S., Camphausen, K., and Tofilon, P. J. (2012). The brain microenvironment preferentially enhances the radioresistance of CD133+ glioblastoma stem-like cells. Neoplasia N. Y. N. 14, 150. doi:10.1593/ neo. 111794

Jamal, M., Rath, B. H., Williams, E. S., Camphausen, K., and Tofilon, P. J. (2010). Microenvironmental regulation of glioblastoma radioresponse. Clin. Cancer Res. 16, 6049-6059. doi:10.1158/1078-0432.CCR-10-2435

Jensen, S. S., Meyer, M., Petterson, S. A., Halle, B., Rosager, A. M., AabergJessen, C., et al. (2016). Establishment and characterization of a tumor stem cell-based glioblastoma invasion model. PLoS ONE 11:e0159746. doi:10.1371/ journal.pone.0159746

Jiguet Jiglaire, C., Baeza-Kallee, N., Denicolaï, E., Barets, D., Metellus, P., Padovani, L., et al. (2014). Ex vivo cultures of glioblastoma in three-dimensional hydrogel maintain the original tumor growth behavior and are suitable for preclinical drug and radiation sensitivity screening. Exp. Cell Res. 321, 99-108. doi:10.1016/j.yexcr.2013.12.010

Jin, S. G., Jeong, Y. I., Jung, S., Ryu, H. H., Jin, Y. H., and Kim, I. Y. (2009). The effect of hyaluronic acid on the invasiveness of malignant glioma cells: comparison of invasion potential at hyaluronic acid hydrogel and matrigel. J. Korean Neurosurg. Soc. 46, 472-478. doi:10.3340/jkns.2009.46.5.472

Kang, K. B., Zhu, C., Wong, Y. L., Gao, Q., Ty, A., and Wong, M. C. (2012). Gefitinib radiosensitizes stem-like glioma cells: inhibition of epidermal growth factor receptor-Akt-DNA-PK signaling, accompanied by inhibition of DNA double-strand break repair. Int. J. Radiat. Oncol. Biol. Phys. 83, e43-e52. doi:10.1016/j.ijrobp.2011.11.037

Kawataki, T., Yamane, T., Naganuma, H., Rousselle, P., Andurén, I., Tryggvason, K., et al. (2007). Laminin isoforms and their integrin receptors in glioma cell migration and invasiveness: evidence for a role of $\alpha 5$-laminin(s) and $\alpha 3 \beta 1$ integrin. Exp. Cell Res. 313, 3819-3831. doi:10.1016/j.yexcr.2007.07.038

Keith, B., Johnson, R. S., and Simon, M. C. (2011). HIF1 $\alpha$ and HIF2 $\alpha$ : sibling rivalry in hypoxic tumor growth and progression. Nat. Rev. Cancer 12, 9-22. doi: $10.1038 / \mathrm{nrc} 3183$

Keunen, O., Johansson, M., Oudin, A., Sanzey, M., Rahim, S. A. A., Fack, F., et al. (2011). Anti-VEGF treatment reduces blood supply and increases tumor cell invasion in glioblastoma. Proc. Natl. Acad. Sci. U.S.A. 108, 3749-3754. doi:10.1073/pnas.1014480108

Kievit, F. M., Florczyk, S. J., Leung, M. C., Veiseh, O., Park, J. O., Disis, M. L., et al. (2010). Chitosan-alginate 3D scaffolds as a mimic of the glioma tumor microenvironment. Biomaterials 31, 5903-5910. doi:10.1016/j.biomaterials.2010. 03.062

Kievit, F. M., Florczyk, S. J., Leung, M. C., Wang, K., Wu, J. D., Silber, J. R., et al. (2014). Proliferation and enrichment of CD133+ glioblastoma cancer stem cells on 3D chitosan-alginate scaffolds. Biomaterials 35, 9137-9143. doi:10.1016/j. biomaterials.2014.07.037

Kievit, F. M., Wang, K., Erickson, A. E., Lan Levengood, S. K., Ellenbogen, R. G., and Zhang, M. (2016). Modeling the tumor microenvironment using chitosan-alginate scaffolds to control the stem-like state of glioblastoma cells. Biomater. Sci. 4, 610-613. doi:10.1039/C5BM00514K

Kim, H.-D., Guo, T. W., Wu, A. P., Wells, A., Gertler, F. B., and Lauffenburger, D. A. (2008). Epidermal growth factor-induced enhancement of glioblastoma cell migration in $3 \mathrm{D}$ arises from an intrinsic increase in speed but an extrinsic matrix- and proteolysis-dependent increase in persistence. Mol. Biol. Cell 19, 4249-4259. doi:10.1091/mbc.E08-05-0501

Kim, Y., and Kumar, S. (2014). CD44-mediated adhesion to hyaluronic acid contributes to mechanosensing and invasive motility. Mol. Cancer Res. 12, 1416-1429. doi:10.1158/1541-7786.MCR-13-0629

Kitai, R., Horita, R., Sato, K., Yoshida, K., Arishima, H., Higashino, Y., et al. (2010). Nestin expression in astrocytic tumors delineates tumor infiltration. Brain Tumor Pathol. 27, 17-21. doi:10.1007/s10014-009-0261-0

Knizetova, P., Ehrmann, J., Hlobilkova, A., Vancova, I., Kalita, O., Kolar, Z., et al. (2008). Autocrine regulation of glioblastoma cell cycle progression, viability and radioresistance through the VEGF-VEGFR2 (KDR) interplay. Cell Cycle Georget. Tex. 7, 2553-2561. doi:10.4161/cc.7.16.6442

Koochekpour, S., Pilkington, G. J., and Merzak, A. (1995). Hyaluronic acid/ $\mathrm{CD} 44 \mathrm{H}$ interaction induces cell detachment and stimulates migration and invasion of human glioma cells in vitro. Int. J. Cancer 63, 450-454. doi:10.1002/ ijc. 2910630325

Kreisl, T. N., Kim, L., Moore, K., Duic, P., Royce, C., Stroud, I., et al. (2008). Fine, phase II trial of single-agent bevacizumab followed by bevacizumab plus irinotecan at tumor progression in recurrent glioblastoma. J. Clin. Oncol. 27, 740-745. doi:10.1200/JCO.2008.16.3055

Lathia, J. D., Gallagher, J., Heddleston, J. M., Wang, J., Eyler, C. E., MacSwords, J., et al. (2010). Integrin alpha 6 regulates glioblastoma stem cells. Cell Stem Cell 6, 421-432. doi:10.1016/j.stem.2010.02.018

Lathia, J. D., Heddleston, J. M., Venere, M., and Rich, J. N. (2011). Deadly teamwork: neural cancer stem cells and the tumor microenvironment. Cell Stem Cell 8, 482-485. doi:10.1016/j.stem.2011.04.013

Lathia, J. D., Li, M., Hall, P. E., Gallagher, J., Hale, J. S., Wu, Q., et al. (2012). Laminin alpha 2 enables glioblastoma stem cell growth. Ann. Neurol. 72, 766-778. doi:10.1002/ana.23674

Lathia, J. D., Mack, S. C., Mulkearns-Hubert, E. E., Valentim, C. L. L., and Rich, J. N. (2015). Cancer stem cells in glioblastoma. Genes Dev. 29, 1203-1217. doi:10.1101/gad.261982.115

Lee, J., Kotliarova, S., Kotliarov, Y., Li, A., Su, Q., Donin, N. M., et al. (2006). Tumor stem cells derived from glioblastomas cultured in bFGF and EGF more closely mirror the phenotype and genotype of primary tumors than do serum-cultured cell lines. Cancer Cell 9, 391-403. doi:10.1016/j.ccr.2006. 03.030

Li, A., Walling, J., Kotliarov, Y., Center, A., Steed, M. E., Ahn, S. J., et al. (2008). Genomic changes and gene expression profiles reveal that established glioma cell lines are poorly representative of primary human gliomas. Mol. Cancer Res. 6, 21-30. doi:10.1158/1541-7786.MCR-07-0280

Li, Q., Lin, H., Wang, O., Qiu, X., Kidambi, S., Deleyrolle, L. P., et al. (2016). Scalable production of glioblastoma tumor-initiating cells in 3 dimension thermoreversible hydrogels. Sci. Rep. 6, 31915. doi:10.1038/srep31915

Li, Z., Bao, S., Wu, Q., Wang, H., Eyler, C., Sathornsumetee, S., et al. (2009). Hypoxia-inducible factors regulate tumorigenic capacity of glioma stem cells. Cancer Cell 15, 501-513. doi:10.1016/j.ccr.2009.03.018

Ligon, K. L., Huillard, E., Mehta, S., Kesari, S., Liu, H., Alberta, J. A., et al. (2007). Olig2-regulated lineage-restricted pathway controls replication competence in neural stem cells and malignant glioma. Neuron 53, 503-517. doi:10.1016/j. neuron.2007.01.009

Liu, G., Yuan, X., Zeng, Z., Tunici, P., Ng, H., Abdulkadir, I. R., et al. (2006). Analysis of gene expression and chemoresistance of CD133+ cancer stem cells in glioblastoma. Mol. Cancer 5, 67. doi:10.1186/1476-4598-5-67

Ljubimova, J. Y., Fujita, M., Khazenzon, N. M., Ljubimov, A. V., and Black, K. L. (2006). Changes in laminin isoforms associated with brain tumor invasion and angiogenesis. Front. Biosci. J. Virtual Libr. 11:81-88. doi:10.2741/1781

Ma, L., Barker, J., Zhou, C., Li, W., Zhang, J., Lin, B., et al. (2012). Towards personalized medicine with a three-dimensional micro-scale perfusion-based two-chamber tissue model system. Biomaterials 33, 4353-4361. doi:10.1016/j. biomaterials.2012.02.054

Ma, N. K. L., Lim, J. K., Leong, M. F., Sandanaraj, E., Ang, B. T., Tang, C., et al. (2016a). Collaboration of 3D context and extracellular matrix in the 
development of glioma stemness in a 3D model. Biomaterials 78, 62-73. doi:10.1016/j.biomaterials.2015.11.031

Mahesparan, R., Read, T.-A., Lund-Johansen, M., Skaftnesmo, K., Bjerkvig, R., and Engebraaten, O. (2003). Expression of extracellular matrix components in a highly infiltrative in vivo glioma model. Acta Neuropathol. 105, 49-57. doi:10.1007/s00401-002-0610-0

Mannino, M., and Chalmers, A. J. (2011). Radioresistance of glioma stem cells: intrinsic characteristic or property of the 'microenvironment-stem cell unit'? Mol. Oncol. 5, 374-386. doi:10.1016/j.molonc.2011.05.001

Matsukado, Y., MacCarty, C. S., and Kernohan, J. W. (1961). The growth of glioblastoma multiforme (astrocytomas, grades 3 and 4 ) in neurosurgical practice. J. Neurosurg. 18, 636-644. doi:10.3171/jns.1961.18.5.0636

Merzak, A., Koocheckpour, S., and Pilkington, G. J. (1994). CD44 mediates human glioma cell adhesion and invasion in vitro. Cancer Res. 54, 3988-3992.

Nakada, M., Nambu, E., Furuyama, N., Yoshida, Y., Takino, T., Hayashi, Y., et al. (2013). Integrin $\alpha 3$ is overexpressed in glioma stem-like cells and promotes invasion. Br. J. Cancer 108, 2516. doi:10.1038/bjc.2013.218

Nakajima, M., Ishimuro, T., Kato, K., Ko, I.-K., Hirata, I., Arima, Y., et al. (2007). Combinatorial protein display for the cell-based screening of biomaterials that direct neural stem cell differentiation. Biomaterials 28, 1048-1060. doi:10.1016/j.biomaterials.2006.10.004

Netti, P. A., Berk, D. A., Swartz, M. A., Grodzinsky, A. J., and Jain, R. K. (2000). Role of extracellular matrix assembly in interstitial transport in solid tumors. Cancer Res. 60, 2497-2503.

Ngo, M. T., and Harley, B. A. (2017). The influence of hyaluronic acid and glioblastoma cell coculture on the formation of endothelial cell networks in gelatin hydrogels. Adv. Healthc. Mater. 6. doi:10.1002/adhm.201700687

Oh, Y., Cha, J., Kang, S.-G., and Kim, P. (2016). A polyethylene glycol-based hydrogel as macroporous scaffold for tumorsphere formation of glioblastoma multiforme. J. Ind. Eng. Chem. 39, 10-15. doi:10.1016/j.jiec.2016.05.012

Omuro, A., and DeAngelis, L. M. (2013). Glioblastoma and other malignant gliomas: a clinical review. JAMA 310, 1842-1850. doi:10.1001/jama.2013.280319

Ortensi, B., Setti, M., Osti, D., and Pelicci, G. (2013). Cancer stem cell contribution to glioblastoma invasiveness. Stem Cell Res. Ther. 4, 18. doi:10.1186/scrt166

Ostrom, Q. T., Gittleman, H., Xu, J., Kromer, C., Wolinsky, Y., Kruchko, C., et al. (2016). CBTRUS statistical report: primary brain and other central nervous system tumors diagnosed in the United States in 2009-2013. Neuro Oncol. 18, v1-v75. doi:10.1093/neuonc/now207

Pàez-Ribes, M., Allen, E., Hudock, J., Takeda, T., Okuyama, H., Viñals, F., et al. (2009). Antiangiogenic therapy elicits malignant progression of tumors to increased local invasion and distant metastasis. Cancer Cell 15, 220-231. doi:10.1016/j.ccr.2009.01.027

Pampaloni, F., Reynaud, E. G., and Stelzer, E. H. K. (2007). The third dimension bridges the gap between cell culture and live tissue. Nat. Rev. Mol. Cell Biol. 8, 839-845. doi:10.1038/nrm2236

Paolillo, M., Serra, M., and Schinelli, S. (2016). Integrins in glioblastoma: still an attractive target? Pharmacol. Res. 113, 55-61. doi:10.1016/j.phrs.2016.08.004

Pathak, A., and Kumar, S. (2012). Independent regulation of tumor cell migration by matrix stiffness and confinement. Proc. Natl. Acad. Sci. U.S.A. 109, 10334-10339. doi:10.1073/pnas.1118073109

Pedron, S., Becka, E., and Harley, B. A. (2015). Spatially gradated hydrogel platform as a 3D engineered tumor microenvironment. Adv. Mater. 27, 1567-1572. doi:10.1002/adma.201404896

Pedron, S., Becka, E., and Harley, B. A. C. (2013). Regulation of glioma cell phenotype in 3D matrices by hyaluronic acid. Biomaterials 34, 7408-7417. doi:10.1016/j.biomaterials.2013.06.024

Pedron, S., Hanselman, J. S., Schroeder, M. A., Sarkaria, J. N., and Harley, B. A. C. (2017). Extracellular hyaluronic acid influences the efficacy of EGFR tyrosine kinase inhibitors in a biomaterial model of glioblastoma. Adv. Healthc. Mater. 6. doi:10.1002/adhm.201700529

Pedron, S., and Harley, B. A. C. (2013). Impact of the biophysical features of a 3D gelatin microenvironment on glioblastoma malignancy. J. Biomed. Mater. Res. A. 101, 3404-3415. doi:10.1002/jbm.a.34637

Petrecca, K., Guiot, M.-C., Panet-Raymond, V., and Souhami, L. (2012). Failure pattern following complete resection plus radiotherapy and temozolomide is at the resection margin in patients with glioblastoma. J. Neurooncol. 111, 19-23. doi:10.1007/s11060-012-0983-4

Pistollato, F., Abbadi, S., Rampazzo, E., Persano, L., Della Puppa, A., Frasson, C., et al. (2010). Intratumoral hypoxic gradient drives stem cells distribution and MGMT expression in glioblastoma. Stem Cells 28, 851-862. doi:10.1002/ stem. 415

Pollard, S. M., Yoshikawa, K., Clarke, I. D., Danovi, D., Stricker, S., Russell, R., et al. (2009). Glioma stem cell lines expanded in adherent culture have tumorspecific phenotypes and are suitable for chemical and genetic screens. Cell Stem Cell 4, 568-580. doi:10.1016/j.stem.2009.03.014

Ponten, J., and Macintyre, E. (1968). Long term culture of normal and neoplastic human glia. Acta Pathol. Microbiol. Scand. 74, 465-486. doi:10.1111/ j.1699-0463.1968.tb03502.x

Rao, S. S., Lannutti, J. J., Viapiano, M. S., Sarkar, A., and Winter, J. O. (2014). Toward 3D biomimetic models to understand the behavior of glioblastoma multiforme cells. Tissue Eng. B Rev. 20, 314-327. doi:10.1089/ten.teb.2013.0227

Rao, S. S., Nelson, M. T., Xue, R., DeJesus, J. K., Viapiano, M. S., Lannutti, J. J., et al. (2013a). Mimicking white matter tract topography using core-shell electrospun nanofibers to examine migration of malignant brain tumors. Biomaterials 34 , 5181-5190. doi:10.1016/j.biomaterials.2013.03.069

Rao, S. S., DeJesus, J., Short, A. R., Otero, J. J., Sarkar, A., and Winter, J. O. (2013b). Glioblastoma behaviors in three-dimensional collagen-hyaluronan composite hydrogels. ACS Appl. Mater. Interfaces 5, 9276-9284. doi:10.1021/ am402097j

Rape, A., Ananthanarayanan, B., and Kumar, S. (2014). Engineering strategies to mimic the glioblastoma microenvironment. Adv. Drug Deliv. Rev. 79-80, 172-183. doi:10.1016/j.addr.2014.08.012

Rape, A. D., and Kumar, S. (2014). A composite hydrogel platform for the dissection of tumor cell migration at tissue interfaces. Biomaterials 35, 8846-8853. doi:10.1016/j.biomaterials.2014.07.003

Rape, A. D., Zibinsky, M., Murthy, N., and Kumar, S. (2015). A synthetic hydrogel for the high-throughput study of cell-ECM interactions. Nat. Commun. 6, 8129 . doi:10.1038/ncomms9129

Rath, B. H., Fair, J. M., Jamal, M., Camphausen, K., and Tofilon, P. J. (2013). Astrocytes Enhance the Invasion Potential of Glioblastoma Stem-Like Cells. PLoS ONE 8:e54752. doi:10.1371/journal.pone.0054752

Rath, B. H., Wahba, A., Camphausen, K., and Tofilon, P. J. (2015). Coculture with astrocytes reduces the radiosensitivity of glioblastoma stem-like cells and identifies additional targets for radiosensitization. Cancer Med. 4, 1705-1716. doi:10.1002/cam4.510

Ricci-Vitiani, L., Pallini, R., Biffoni, M., Todaro, M., Invernici, G., Cenci, T., et al. (2010). Tumour vascularization via endothelial differentiation of glioblastoma stem-like cells. Nature 468, 824-828. doi:10.1038/nature09557

Rich, J. N. (2007). Cancer stem cells in radiation resistance. Cancer Res. 67, 8980-8984. doi:10.1158/0008-5472.CAN-07-0895

Ruiz-Ontañon, P., Orgaz, J. L., Aldaz, B., Elosegui-Artola, A., Martino, J., Berciano, M. T., et al. (2013). Cellular plasticity confers migratory and invasive advantages to a population of glioblastoma-initiating cells that infiltrate peritumoral tissue. Stem Cells 31, 1075-1085. doi:10.1002/stem.1349

Safa, A. R., Saadatzadeh, M. R., Cohen-Gadol, A. A., Pollok, K. E., and BijangiVishehsaraei, K. (2015). Glioblastoma stem cells (GSCs) epigenetic plasticity and interconversion between differentiated non-GSCs and GSCs. Genes Dis. 2, 152-163. doi:10.1016/j.gendis.2015.02.001

Saha, K., Keung, A. J., Irwin, E. F., Li, Y., Little, L., Schaffer, D. V., et al. (2008). Substrate modulus directs neural stem cell behavior. Biophys. J. 95, 4426-4438. doi:10.1529/biophysj.108.132217

Sanai, N., Alvarez-Buylla, A., and Berger, M. S. (2005). Neural stem cells and the origin of gliomas. N. Engl. J. Med. 353, 811-822. doi:10.1056/NEJMra043666

Sanai, N., Polley, M.-Y., McDermott, M. W., Parsa, A. T., and Berger, M. S. (2011). An extent of resection threshold for newly diagnosed glioblastomas. J. Neurosurg. 115, 3-8. doi:10.3171/2011.2.JNS10998

Sarkar, S., Nuttall, R. K., Liu, S., Edwards, D. R., and Yong, V. W. (2006). Tenascin-C stimulates glioma cell invasion through matrix metalloproteinase-12. Cancer Res. 66, 11771-11780. doi:10.1158/0008-5472.CAN-05-0470

Schonberg, D. L., Lubelski, D., Miller, T. E., and Rich, J. N. (2014). Brain tumor stem cells: molecular characteristics and their impact on therapy. Mol. Aspects Med. 39, 82-101. doi:10.1016/j.mam.2013.06.004

Seidel, S., Garvalov, B. K., Wirta, V., von Stechow, L., Schänzer, A., Meletis, K., et al. (2010). A hypoxic niche regulates glioblastoma stem cells through hypoxia inducible factor $2 \alpha$. Brain 133, 983-995. doi:10.1093/brain/awq042

Shen, Q., Goderie, S. K., Jin, L., Karanth, N., Sun, Y., Abramova, N., et al. (2004). Endothelial cells stimulate self-renewal and expand neurogenesis of neural stem cells. Science 304, 1338-1340. doi:10.1126/science.1095505 
Singh, S., Hawkins, C., Clarke, I. D., Squire, J. A., Bayani, J., Hide, T., et al. (2004). Identification of human brain tumour initiating cells. Nature 432, 393-396. doi:10.1038/nature03031

Singh, S. K., Clarke, I. D., Terasaki, M., Bonn, V. E., Hawkins, C., Squire, J., et al. (2003). Identification of a cancer stem cell in human brain tumors. Cancer Res. 63, 5821-5828.

Soda, Y., Myskiw, C., Rommel, A., and Verma, I. M. (2013). Mechanisms of neovascularization and resistance to anti-angiogenic therapies in glioblastoma multiforme. J. Mol. Med. Berl. Ger. 91, 439-448. doi:10.1007/s00109-013-1019-z

Soeda, A., Park, M., Lee, D., Mintz, A., Androutsellis-Theotokis, A., McKay, R. D., et al. (2009). Hypoxia promotes expansion of the CD133-positive glioma stem cells through activation of HIF-1 $\alpha$. Oncogene 28, 3949-3959. doi:10.1038/ onc.2009.252

Soen, Y., Mori, A., Palmer, T. D., and Brown, P. O. (2006). Exploring the regulation of human neural precursor cell differentiation using arrays of signaling microenvironments. Mol. Syst. Biol. 2, 37. doi:10.1038/msb4100076

Son, M. J., Woolard, K., Nam, D.-H., Lee, J., and Fine, H. A. (2009). SSEA-1 is an enrichment marker for tumor-initiating cells in human glioblastoma. Cell Stem Cell 4, 440-452. doi:10.1016/j.stem.2009.03.003

Sottoriva, A., Spiteri, I., Piccirillo, S. G. M., Touloumis, A., Collins, V.P., Marioni, J. C., et al. (2013). Intratumor heterogeneity in human glioblastoma reflects cancer evolutionary dynamics. Proc. Natl. Acad. Sci. U.S.A. 110, 4009-4014. doi:10.1073/pnas.1219747110

Strojnik, T., Røsland, G. V., Sakariassen, P. O., Kavalar, R., and Lah, T. (2007). Neural stem cell markers, nestin and musashi proteins, in the progression of human glioma: correlation of nestin with prognosis of patient survival. Surg. Neurol. 68, 133-143. doi:10.1016/j.surneu.2006.10.050

Stupp, R., Hegi, M. E., Mason, W. P., van den Bent, M. J., Taphoorn, M. J., Janzer, R. C., et al. (2009). Effects of radiotherapy with concomitant and adjuvant temozolomide versus radiotherapy alone on survival in glioblastoma in a randomised phase III study: 5-year analysis of the EORTC-NCIC trial. Lancet Oncol. 10, 459-466. doi:10.1016/S1470-2045(09)70025-7

Tamaki, M., McDonald, W., Amberger, V. R., Moore, E., and Del Maestro, R. F. (1997). Implantation of C6 astrocytoma spheroid into collagen type I gels: invasive, proliferative, and enzymatic characterizations. J. Neurosurg. 87, 602-609. doi:10.3171/jns.1997.87.4.0602

Tan, B. T., Park, C. Y., Ailles, L. E., and Weissman, I. L. (2006). The cancer stem cell hypothesis: a work in progress. Lab. Invest. 86, 1203-1207. doi:10.1038/ labinvest. 3700488

Teixeira, A. I., Duckworth, J. K., and Hermanson, O. (2007). Getting the right stuff: controlling neural stem cell state and fate in vivo and in vitro with biomaterials. Cell Res. Lond. 17, 56-61. doi:10.1038/sj.cr.7310141

Ulrich, T. A., de Juan Pardo, E. M., and Kumar, S. (2009). The mechanical rigidity of the extracellular matrix regulates the structure, motility, and proliferation of glioma cells. Cancer Res. 69, 4167-4174. doi:10.1158/0008-5472.CAN-08-4859

Ulrich, T. A., Jain, A., Tanner, K., MacKay, J. L., and Kumar, S. (2010). Probing cellular mechanobiology in three-dimensional culture with collagen-agarose matrices. Biomaterials 31, 1875-1884. doi:10.1016/j.biomaterials.2009.10.047

Umesh, V., Rape, A. D., Ulrich, T. A., and Kumar, S. (2014). Microenvironmental stiffness enhances glioma cell proliferation by stimulating epidermal growth factor receptor signaling. PLOS ONE 9:e101771. doi:10.1371/journal.pone. 0101771

Venere, M., Fine, H. A., Dirks, P. B., and Rich, J. N. (2011). Cancer stem cells in gliomas: identifying and understanding the apex cell in cancer's hierarchy. Glia 59, 1148-1154. doi:10.1002/glia.21185

Venugopal, C., McFarlane, N. M., Nolte, S., Manoranjan, B., and Singh, S. K. (2012). Processing of primary brain tumor tissue for stem cell assays and flow sorting. J. Vis. Exp. 67. doi:10.3791/4111

Verhaak, R. G. W., Hoadley, K. A., Purdom, E., Wang, V., Qi, Y., Wilkerson, M. D., et al. (2010). Integrated genomic analysis identifies clinically relevant subtypes of glioblastoma characterized by abnormalities in PDGFRA, IDH1, EGFR, and NF1. Cancer Cell 17, 98-110. doi:10.1016/j.ccr.2009.12.020

Wang, C., Tong, X., and Yang, F. (2014). Bioengineered 3D brain tumor model to elucidate the effects of matrix stiffness on glioblastoma cell behavior using PEG-based hydrogels. Mol. Pharm. 11, 2115-2125. doi:10.1021/mp5000828

Wang, K., Kievit, F. M., Erickson, A. E., Silber, J. R., Ellenbogen, R. G., and Zhang, M. (2016). Culture on 3D chitosan-hyaluronic acid scaffolds enhances stem cell marker expression and drug resistance in human glioblastoma cancer stem cells. Adv. Healthc. Mater. 5, 3173-3181. doi:10.1002/adhm.201600684

Wang, R., Chadalavada, K., Wilshire, J., Kowalik, U., Hovinga, K. E., Geber, A., et al. (2010a). Glioblastoma stem-like cells give rise to tumour endothelium. Nature 468, 829-833. doi:10.1038/nature09624

Wang, J., Wakeman, T. P., Lathia, J. D., Hjelmeland, A. B., Wang, X.-F., White, R. R., et al. (2010b). Notch promotes radioresistance of glioma stem cells. Stem Cells 28, 17-28. doi:10.1002/stem.261

Wiranowska, M., and Plaas, A. (2008). Cytokines and extracellular matrix remodeling in the central nervous system. NeuroImmune Biol. 6, 167-197. doi:10.1016/S1567-7443(07)10009-0

Wiranowska, M., and Rojiani, M. V. (2011). Extracellular Matrix Microenvironment in Glioma Progression. INTECH Open Access Publisher. Available at: https:// cdn.intechopen.com/pdfs-wm/22475.pdf.

Wong, S. Y., Ulrich, T. A., Deleyrolle, L. P., MacKay, J. L., Lin, J.-M. G., Martuscello, R. T., et al. (2015). Constitutive activation of myosin-dependent contractility sensitizes glioma tumor-initiating cells to mechanical inputs and reduces tissue invasion. Cancer Res. 75, 1113-1122. doi:10.1158/0008-5472.CAN-13-3426

Woolard, K., and Fine, H. A. (2009). Glioma stem cells: better flat than round. Cell Stem Cell 4, 466-467. doi:10.1016/j.stem.2009.05.013

Xiao, W., Sohrabi, A., and Seidlits, S. K. (2017). Integrating the glioblastoma microenvironment into engineered experimental models. Future Sci. OA 3, FSO189. doi:10.4155/fsoa-2016-0094

Yang, F., Murugan, R., Wang, S., and Ramakrishna, S. (2005). Electrospinning of nano/micro scale poly(l-lactic acid) aligned fibers and their potential in neural tissue engineering. Biomaterials 26, 2603-2610. doi:10.1016/j. biomaterials.2004.06.051

Yang, M.-Y., Chiao, M.-T., Lee, H.-T., Chen, C.-M., Yang, Y.-C., Shen, C.-C., et al. (2014). An innovative three-dimensional gelatin foam culture system for improved study of glioblastoma stem cell behavior. J. Biomed. Mater. Res. B Appl. Biomater. 103, 618-628. doi:10.1002/jbm.b.33214

Yang, Y., Motte, S., and Kaufman, L. J. (2010). Pore size variable type I collagen gels and their interaction with glioma cells. Biomaterials 31, 5678-5688. doi:10.1016/j.biomaterials.2010.03.039

Zeng, L., Zhao, Y., Ouyang, T., Zhao, T., Zhang, S., Chen, J., et al. (2016). Label-retaining assay enriches tumor-initiating cells in glioblastoma spheres cultivated in serum-free medium. Oncol. Lett. 12, 815-824. doi:10.3892/ ol.2016.4690

Zhang, S., Xie, R., Wan, F., Ye, F., Guo, D., and Lei, T. (2013). Identification of U251 glioma stem cells and their heterogeneous stem-like phenotypes. Oncol. Lett. 6, 1649-1655. doi:10.3892/ol.2013.1623

Conflict of Interest Statement: The authors declare that the research was conducted in the absence of any commercial or financial relationships that could be construed as a potential conflict of interest.

Copyright (C) 2018 Heffernan and Sirianni. This is an open-access article distributed under the terms of the Creative Commons Attribution License (CC BY). The use, distribution or reproduction in other forums is permitted, provided the original author(s) and the copyright owner are credited and that the original publication in this journal is cited, in accordance with accepted academic practice. No use, distribution or reproduction is permitted which does not comply with these terms. 